Research paper

\title{
Synthesis and in-depth studies on the anticancer activity of novel palladacyclopentadienyl complexes stabilized by $\mathrm{N}$-Heterocyclic carbene ligands
}

\author{
Thomas Scattolin ${ }^{\text {a }}$, Isabella Caligiuri ${ }^{\mathrm{b}}$, Nayla Mouawad ${ }^{\mathrm{b}}$, Maguie El Boustani ${ }^{\mathrm{b}}$, \\ Nicola Demitri ${ }^{\text {c }}$, Flavio Rizzolio ${ }^{\text {a, b }}$, Fabiano Visentin ${ }^{\text {a, * }}$ \\ a Dipartimento di Scienze Molecolari e Nanosistemi, Università Ca' Foscari, Campus Scientifico Via Torino 155, 30174, Venezia-Mestre, Italy \\ ${ }^{\mathrm{b}}$ Pathology Unit, IRCCS CRO Aviano-National Cancer Institute, 33081, Aviano, Italy \\ ${ }^{c}$ Elettra - Sincrotrone Trieste, S.S. 14 Km 163.5 in Area Science Park, 34149, Basovizza, Trieste, Italy
}

\section{A R T I C L E I N F O}

\section{Article history:}

Received 14 May 2019

Received in revised form

16 May 2019

Accepted 22 June 2019

Available online 22 June 2019

\section{Keywords:}

Organometallic anticancer agents

Palladacyclopentadienyl compounds

Ovarian cancer

$\mathrm{N}$-Heterocyclic carbenes

DNA damage

\begin{abstract}
A B S T R A C T
New palladacyclopentadienyl complexes with bis-N-heterocyclic carbenes as spectator ligands have been synthesized and exhaustively characterized. The crystal structure of complex 1a has been also determined by X-ray diffraction analysis. Their in vitro cytotoxicity and that of other palladacyclopentadienyl derivatives coordinating different ancillary ligands has been determined against different cancer cell lines. Many complexes have shown an antiproliferative activity toward tumor cells often definitely better than cisplatin, whereas they have resulted practically inactive against the non-cancer MRC- 5 cell line. The mechanism of action of bis-NHC derivative 1a, particularly active against ovarian cancer cell lines was studied in depth. Through a longitudinally analysis, it is shown that compound $\mathbf{1 a}$ induces apoptosis via DNA damage and release of cytochrome $C$. We propose compound $\mathbf{1 a}$ as a powerful and specific drug for the therapy of a deadly disease such as high grade serous ovarian cancer.
\end{abstract}

๑) 2019 Elsevier Masson SAS. All rights reserved.

\section{Introduction}

Since the discovery of cisplatin, a successful chemotherapeutic drug approved by the FDA in 1978 , a great number of platinum complexes has been synthesized in hopes of reducing the side effects and the resistance mechanisms observed for some types of tumors treated with cisplatin-based therapies [1].

Unfortunately, the second and third generation of platinum chemotherapy drugs (i.e. carboplatin and oxaliplatin) have retained some contraindications and moreover have proven in many cases inactive or poorly active against cisplatin-resistant neoplasms [2]. For this reason, many research groups have focused their efforts on finding new anticancer agents based on other transition metals [3]. Among the non-platinum derivatives, palladium complexes have received an increasing attention for their good antiproliferative effects, generally associated with different mechanisms of action as compared to cisplatin and analogues. Furthermore, palladium compounds have the advantage of being generally less expensive

\footnotetext{
* Corresponding author.

E-mail address: fvise@unive.it (F. Visentin).
}

and more water-soluble than platinum homologues [4]. On the other hand, a potential limitation is represented by their high rate of hydrolysis, and the elevated lability of the coordinated ligands is often associated with the sequestration of the metal by biomolecules containing soft donating atoms, mainly proteins and derivatives $[4,5]$. This feature increases their general toxicity and reduces the possibility that the drug could selectively reach the predicted target [6]. This critical point suggests that a careful choice of the ligands coordinating the metal centre may play a key role in the design of palladium anticancer compounds. In this regard, it has recently been shown that ligands firmly anchored to palladium reduce or eliminate the hydrolysis problems [7].

$\mathrm{N}$-heterocyclic carbenes (NHCs) represent a class of ligands able to satisfy this important requirement [8] and thus, beside homogeneous catalysis [9], have found numerous applications in the biomedical field, mostly in the synthesis of antitumor $[7,10]$ and antimicrobial compounds [11].

The synthesis of Pd (II) complexes stabilized by NHCs deriving from natural xanthine bases and containing the $\eta^{3}$-allyl or palladacyclopentadienyl organometallic fragments has recently been reported by our group [12]. These works were justified by the fact 
that the biological activity of complexes bearing such fragments was practically unexplored, whereas their reactivity and applications in the field of catalysis are well known [13,14].

The antiproliferative and proapoptotic activity tests carried out on human ovarian cancer lines have shown that some of the synthesized complexes exhibit higher activity than cisplatin both on cisplatin-sensitive and cisplatin-resistant lines. At the same time, these compounds are poorly active or inactive towards normal cells (fibroblasts).

Encouraged by these first results, in the present paper we propose an in-depth study of the antitumor activity of the palladacyclopentadienyl complexes reported in Chart 1 against different types of human cancer cells.

The palladacyclopentadienyl framework is a particularly stable structure as proved by numerous studies on the reactivity of complexes containing this organometallic function, carried out by our research group [15]. In particular, it was shown that these species are quickly attacked only by strong oxidants such as halogens, which are not usually present in biological environment. Therefore, if the palladacyclopentadienyl motif is combined with strong spectator ligands may be possible to obtain very stable complexes that could behave like structural compounds in the cellular environment.

In a recent paper published by Che and co-workers, cyclometallated Pd (II) complexes with different spectator ligands have been reported. For these complexes, the antiproliferative activity has been found to depend strongly on the type of spectator ligand. The most effective complexes are those coordinating a $\mathrm{N}$-heterocyclic carbene ligand; they are stable in the presence of biological thiols (i.e. glutathione) and act as structural compounds mainly by altering the mitochondrial membrane of the cancer cells [7e].

On the basis of these facts, we report the synthesis of yet unpublished palladacyclopentadienyl compounds stabilized by chelating bisNHCs (1a-b) [16].

The antiproliferative activity against fibroblasts (normal cells) and different tumor cell lines is compared with that of complexes containing other spectator ligands $(\mathbf{2}-\mathbf{6})$, previously prepared and described by our research group $[12,17]$.

The panel of human tumor cell lines tested includes ovarian carcinomas (A2780 and OVCAR5), lung cancer (A549), malignant melanoma (A375) and colon cancer (DLD1).

Finally, for the complex 1a we will also propose the results of specific biological tests aimed at identifying the main biological target and defining the most probable mechanism of action.

\section{Results and discussion}

\subsection{Synthesis of palladacyclopentadienyl complexes bearing chelating bisNHCs (1a-b)}

Preliminary tests have highlighted that only the complexes with benzyl substituents in the wing tip position can be selectively obtained. As a matter of fact, when the less cumbersome (i.e. methyl) or bulkier (i.e. mesityl) substituents are involved, some byproducts, which are difficult to identify and separate, are present in reaction mixtures in addition to the compounds of interest.

Compounds 1a-b were therefore synthesized by reacting the complexes Aga-b with the polymeric precursor $\left[\mathrm{PdC}_{4}(\mathrm{COOMe})_{4}\right]_{\mathrm{n}}$ (Scheme 1), which were prepared according to the published procedures [18,19].

Transmetallation proceeds in very good yield and the resulting species were all stable in air and in solution.

The characterization of such compounds was performed by NMR, IR and elemental analysis. In the ${ }^{1} \mathrm{H}$ spectra (Figs. S1-2) it is possible to observe:

a) two singlets ascribable to two different pairs of $\mathrm{OCH}_{3}$ groups within 3 and $4 \mathrm{ppm}$;

b) an $\mathrm{AB}$ system $(J \approx 15 \mathrm{~Hz})$, between 5 and $6 \mathrm{ppm}$, ascribable to the methylene protons of the benzyl substituents;

c) an $\mathrm{AB}$ system $(J=13.6 \mathrm{~Hz})$, deriving from the protons of the methylene bridge (for complex 1a) within 6 and $6.5 \mathrm{ppm}$;

d) two multiplets, between 4 and $5.5 \mathrm{ppm}$, concerning the protons of the ethylene bridge (for complex 1b).

In the ${ }^{13} \mathrm{C}\left\{\mathrm{H}^{1}\right\}$-NMR spectra (Figs. $\mathrm{S} 3-4$ ), the signals ascribable to the two different pairs of $\underline{\mathrm{C}}\left(\mathrm{COOCH}_{3}\right)$ groups are detected in addition to the peak of the carbenic carbon at about $180 \mathrm{ppm}$. A selection of the bi-dimensional NMR spectra (HSQC and HMBC) is reported in Figs. S5-6.

Finally, the presence of the carbonyl groups of the cyclopalladated fragment is testified by the intense stretching bands between 1730 and $1680 \mathrm{~cm}^{-1}$ in the IR spectra (Fig. S7).

\subsection{X-ray crystal structure determination of $\mathbf{1 a}$}

The solid-state structure of derivative 1a was confirmed by Xray diffraction. Its crystalline form contains one crystallographically independent palladium complex showing a square planar coordination (Fig. 1 and S8, Table S2). Crystal packing of 1a shows hydrophobic contacts among neighbour molecules, involving $\mathrm{CH} \cdots \pi$, and weak intermolecular $\pi \cdots \pi$ interactions among neighbour phenyl sidechains of the $N$-heterocyclic carbene ligands (Fig. 1).

The metal bound ligands adopt conformations equivalent to those of the complexes with chelating bis( $N$-heterocyclic carbene) ligands or palladacyclopentadienyl fragments reported in Table S3. Both CCDC 1844914 [18] and CCDC 1881566 [12b] show almost overlapping conformations (R.M.S.D. of common atoms shared by the models is $\sim 0.60 \AA$, Fig. S9). Notably, the NHC scaffold varies its angle with respect to the Pd coordination plane as a function of the bulkiness of the partner bidentate ligand bound. The mutual angle between the NHC mean plane and Pd coordination plane increases

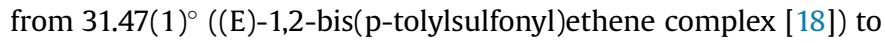
$60.62(7)^{\circ}(1 \mathbf{a})$, due to the increased bite angle and steric hindrance

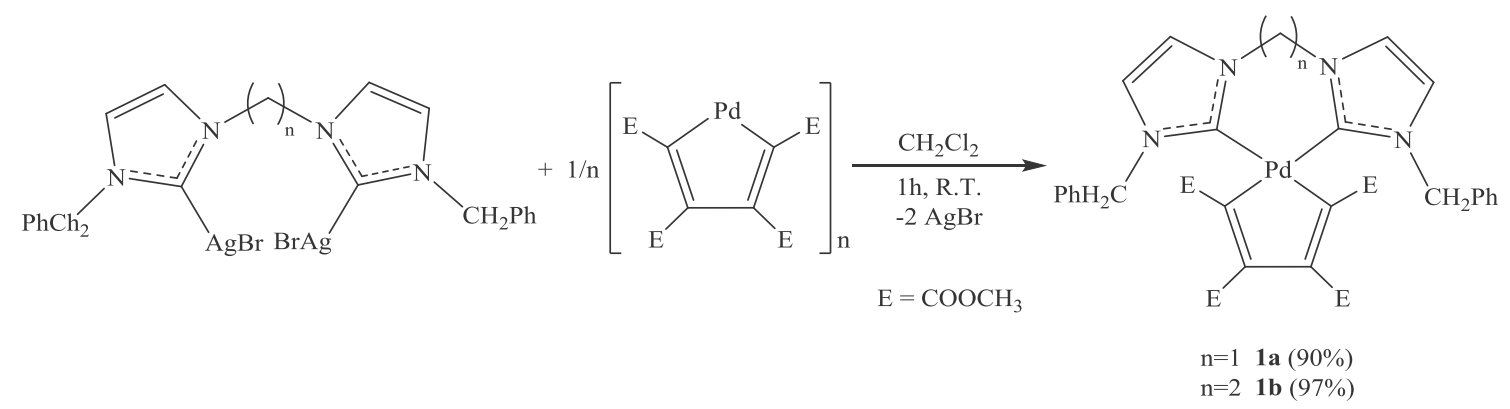

Scheme 1. Synthesis of complexes 1a-b. 


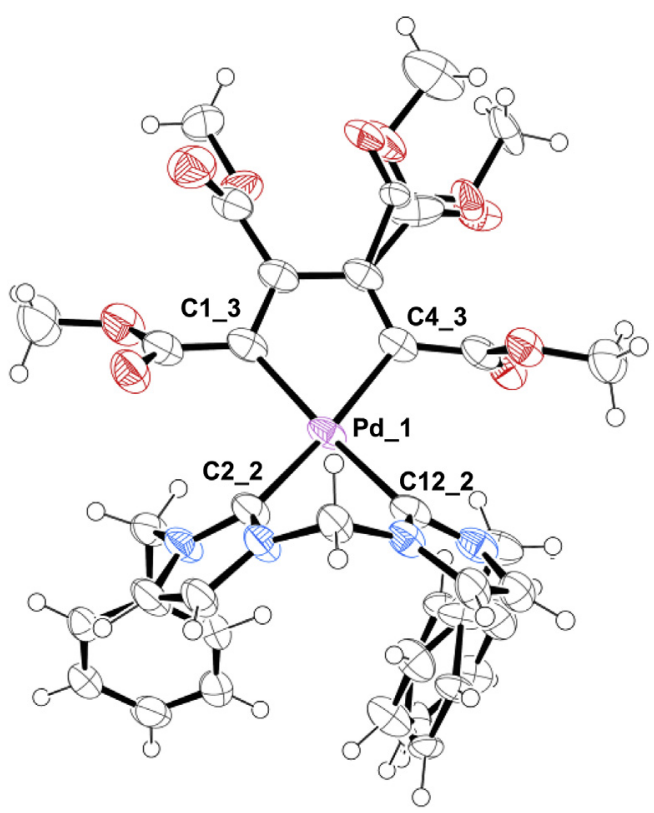

Fig. 1. Ellipsoid representation of 1a crystals ASU contents ( $50 \%$ probability). Atom labels in use for Pd coordination sphere are reported.

of (C-COOR $)_{4}$ carbomethoxy sidechains in 1a (Figs. S10A-B). This steric effect is consistent when 1a is compared with the less bulky NHC ligand [(1-(2-pyridyl)methylene-3-methyl]imidazolyl-2-ene), which leads to a less pronounced angle of 56.23(6) $)^{\circ}$ (CCDC 1059980 [15c], Fig. S10D). Furthermore, when the NHC imidazolyl ring is free to rotate $(\mathrm{C})$ it stays almost perpendicular to the palladium coordination sphere $\left(83.48(9)^{\circ}\right.$ in CCDC 1059981 [15c], Fig. S10C). It can also be noticed that the carbene conformation in 1a distorts the palladium coordination sphere pushing the Pd atom out of its coordination plane (distance of Pd from the plane increases from $0.018(1) \AA$ in CCDC 1059980 [15c] or 0.033(1) $\AA$ in the ethene variant [18] to $0.1378(2) \AA$ in $\mathbf{1 a})$.

\subsection{In vitro antiproliferative activities}

The antiproliferative activity of all complexes reported in Chart 1 was evaluated on a panel of different human tumor cell lines (ovarian cancers OVCAR5 and A2780 with its cisplatin resistant clone A2780cis, lung cancer A549, malignant melanoma A375 and colon adenocarcinoma DLD1) and MRC-5 normal cells (human fibroblasts). Preliminarily, the stability of all compounds was checked in 1:1 DMSO- $d_{6} / \mathrm{D}_{2} \mathrm{O}$ solution (4a-b, 3c-d, and $\mathbf{5 a - b}$ and $\mathbf{6}$ ) or DMSO- $d_{6}$ (1a-b, 2a-c and 3a-b) by NMR spectroscopy: after $48 \mathrm{~h}$ at room temperature no noticeable degradation was observed.

The half inhibitory concentrations $\left(\mathrm{IC}_{50}\right)$ induced by palladium complexes are summarized in Table 1 together with the corresponding values of cisplatin (positive control).

From a first analysis of the data, it is evident that a good number of the tested complexes show a remarkable cytotoxicity against tumor cell lines, often better than cisplatin, whereas they proved almost inactive toward the MRC-5 normal cells $\left(\mathrm{IC}_{50}>100 \mu \mathrm{M}\right)$, with the only exceptions of $\mathbf{3 a}$ and $\mathbf{3 c}$.

In more detail, it is possible to observe that: a) all complexes bearing at least one $N$-heterocyclic carbene ligand $(\mathbf{1 - 4})$ are significantly active against ovarian cancer and malignant melanoma cell lines. Complexes $\mathbf{1 a}$ and $\mathbf{1 b}$, (with the chelating bis-NHCs ligands) are the most efficient species, with $\mathrm{IC}_{50}$ values always lower than cisplatin; b) all complexes coordinating at least one monodentate $\mathrm{N}$-heterocyclic carbene (2 and $\mathbf{3}$ with the only exception of $\mathbf{3 b}$ ) show a remarkable cytotoxicity towards DLD1 colon adenocarcinoma cells. Among them, compound 2a presents an $\mathrm{IC}_{50}$ value about 200 times lower than cisplatin; c) the complexes with mixed NHC and isocyanide or phosphane ligands (3) exhibit a marked antiproliferative activity versus A549 lung cancer, with compounds $\mathbf{3 b}$ and $\mathbf{3 d}$ standing out for their particularly low $\mathrm{IC}_{50}$ values; d) the palladacyclopentadienyl complexes with chelating phosphoquinoline or thioquinoline/thiopyridine ligands are poorly active or inactive toward all investigated cancer cell lines.

These results highlight that in the group of examined compounds it is always possible to get one or more complexes with a marked and selective antiproliferative activity against one of the specific cancer cell lines investigated. The presence of at least one $\mathrm{N}$-heterocyclic carbene as ancillary ligand is a necessary requisite for obtaining this result.

\subsection{Reactivity of complexes with GSH}

The stability of palladacyclopentadienyl derivatives in the presence of the biological reductant thiol glutathione (GSH) was also examined. The tests carried out in DMSO- $d_{6}$ or DMSO- $d_{6} / \mathrm{D}_{2} \mathrm{O}$

Table 1

Effects of the Pd-complexes on the viability of several cancer and normal cells lines ${ }^{\text {a }}$

\begin{tabular}{|c|c|c|c|c|c|c|c|}
\hline \multirow[t]{2}{*}{ Complex } & \multicolumn{7}{|l|}{$\mathrm{IC}_{50}(\mu \mathrm{M})$} \\
\hline & A2780 & A2780cis & OVCAR5 & A549 & A375 & DLD1 & MRC-5 \\
\hline Cisplatin & $0.81 \pm 0.06$ & $43 \pm 1$ & $0.84 \pm 0.04$ & $6 \pm 3$ & $4.7 \pm 0.4$ & $19 \pm 4$ & $14 \pm 1$ \\
\hline $1 \mathrm{a}$ & $0.039 \pm 0.006$ & $2.8 \pm 0.6$ & $0.30 \pm 0.07$ & $>100$ & $0.3 \pm 0.1$ & $>100$ & $>100$ \\
\hline 1b & $0.46 \pm 0.05$ & $7 \pm 5$ & $0.49 \pm 0.09$ & $>100$ & $2.5 \pm 0.7$ & $>100$ & $>100$ \\
\hline $2 a$ & $46 \pm 11$ & $31 \pm 1$ & $0.13 \pm 0.07$ & $>100$ & $19 \pm 16$ & $0.10 \pm 0.09$ & $>100$ \\
\hline $2 \mathbf{b}$ & $2.6 \pm 0.4$ & $12 \pm 3$ & $7 \pm 2$ & $>100$ & $9 \pm 5$ & $7 \pm 1$ & $>100$ \\
\hline $2 c$ & $0.58 \pm 0.03$ & $4.4 \pm 0.3$ & $13 \pm 3$ & $>100$ & $10.2 \pm 0.1$ & $34 \pm 15$ & $>100$ \\
\hline $3 \mathbf{a}$ & $0.33 \pm 0.08$ & $1.3 \pm 0.4$ & $1.2 \pm 0.4$ & $17 \pm 9$ & $4.4 \pm 0.3$ & $4.7 \pm 0.9$ & $4.7 \pm 0.2$ \\
\hline 3b & $3.9 \pm 0.6$ & $3.4 \pm 0.8$ & $1 \pm 1$ & $0.05 \pm 0.02$ & $29 \pm 7$ & $>100$ & $>100$ \\
\hline $3 c$ & $1.0 \pm 0.2$ & $0.6 \pm 0.2$ & $3.5 \pm 0.4$ & $2.8 \pm 0.2$ & $5.6 \pm 0.5$ & $5.2 \pm 0.7$ & $4.8 \pm 0.2$ \\
\hline 3d & $2.6 \pm 0.4$ & $3.9 \pm 0.6$ & $13 \pm 2$ & $0.24 \pm 0.09$ & $4.9 \pm 0.9$ & $7 \pm 2$ & $>100$ \\
\hline $4 a$ & $3.7 \pm 0.2$ & $4.4 \pm 0.9$ & $1.79 \pm 0.06$ & $>100$ & $2 \pm 2$ & $>100$ & $>100$ \\
\hline $4 b$ & $2.7 \pm 0.2$ & $>100$ & $52 \pm 26$ & $>100$ & $24 \pm 9$ & $>100$ & $>100$ \\
\hline $5 \mathbf{a}$ & $16 \pm 5$ & $>100$ & $>100$ & $>100$ & $37 \pm 3$ & $>100$ & $>100$ \\
\hline $5 \mathbf{b}$ & $98 \pm 49$ & $>100$ & $>100$ & $>100$ & $>100$ & $>100$ & $>100$ \\
\hline 6 & $10 \pm 2$ & $28 \pm 3$ & $>100$ & $>100$ & $>100$ & $>100$ & $>100$ \\
\hline
\end{tabular}

a Data after $96 \mathrm{~h}$ of incubation. Stock solutions in DMSO for all complexes; stock solutions in $\mathrm{H}_{2} \mathrm{O}$ for cisplatin. A2780 and OVCAR5 (cisplatin-sensitive ovarian cancer cells),

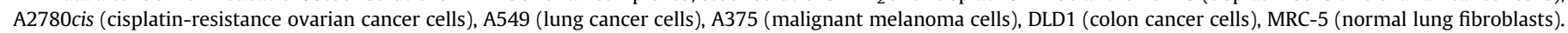


$(1: 1 \mathrm{v} / \mathrm{v})$ adding a 5 -fold excess of glutathione to a $2 \mathrm{mM}$ solution of each complex were followed by ${ }^{1} \mathrm{H}$ NMR spectroscopy. It was observed that: a) the complexes containing a chelating or two monodentate carbene ligands (1-2), as well as the mixed NHC/DIC derivatives (3c-d), were stable since no significant changes of their spectra had been recorded for $48 \mathrm{~h}$; b) in the mixed $\mathrm{NHC} / \mathrm{PPh}_{3}$ and phosphoquinoline complexes (3a-b and $\mathbf{5 a}$ respectively) the phosphine ligands were completely released in solution within $24 \mathrm{~h}$ and promptly converted into the respective phosphine oxides; c) the complexes coordinating NHC-thioether (4a) and NHCpyridine (4b) underwent the labile arm opening immediately after addition of GSH: the system did not evolve further and the carbene remained firmly anchored to the metal centre; d) in the complexes bearing thioquinoline (5b) and thiopyridine $(\mathbf{6})$ the bidentate ligands were quickly replaced by glutathione.

\subsection{Biological activity}

Complex 1a was selected for mechanistic studies since it is the most potent on ovarian cancer cell lines with no activity on normal cells. To strengthen the data, the biological experiments were carried out by a kinetic analysis at different timepoints. With this approach it is possible to determine the primary biological effects generated directly by the palladium compound.

One of the possible mechanisms related to organometallic anticancer compounds involves an interaction with DNA and its consequent damage [20]. To test this mechanism, as a sensitive marker for DNA double-strand breaks, the phosphorylation of Ser139 of Histone H2A.X was evaluated. After DNA damage, the phosphorylation at Ser139 helps to recruit the DNA repair machine and apoptotic proteins to dispatch their function [21].

Cells were treated with 40,80, $320 \mathrm{nM}$ of complex $1 \mathrm{a}$ for 3, 6 and $12 \mathrm{~h}$. As shown in Fig. 2, after $3 \mathrm{~h}$ the phosphorylation of Ser139Histone H2A.X starts to accumulate in the nucleus (dotted green, arrows). The complex was active already at $40 \mathrm{nM}$, which corresponds to the $\mathrm{IC}_{50}$ value. In the immunofluorescence images, it appears as foci in the nucleus. Cisplatin was utilized as positive control at $10 \mu \mathrm{M}$.

DNA integrity was analyzed by flow cytometry. SubG1 fraction was considered as marker of DNA fragmentation. As clearly observable in Fig. 3, the subG1 fraction of cells treated with compound $\mathbf{1 a}$ is dose and time dependent.

At molecular level, cells could activate the apoptotic pathway by inducing the release of cytochrome $\mathrm{C}$, a conserved electrontransport protein which is part of the respiratory chain of mitochondria. This early event was studied by immunofluorescence. Cells were treated as in Fig. 4.

After 6-12 h, A2780 cells treated with 1a release cytochrome C from mitochondria to cytoplasm and appear as diffuse cytoplasmic staining pattern (Fig. 4). After the release of cytochrome C, cells activate the caspase cascade to induce apoptosis [22]. The induction of caspase 3/7 was assessed at different time points and concentrations of compound 1a as shown in immunofluorescence experiments (Fig. 5). Starting from $40 \mathrm{nM}$ of complex, cells displayed a high level of caspase 3/7 compared to untreated cells.

\section{Conclusions}

In this paper, we have described the synthesis and the complete structural characterization of novel palladacyclopentadienyl

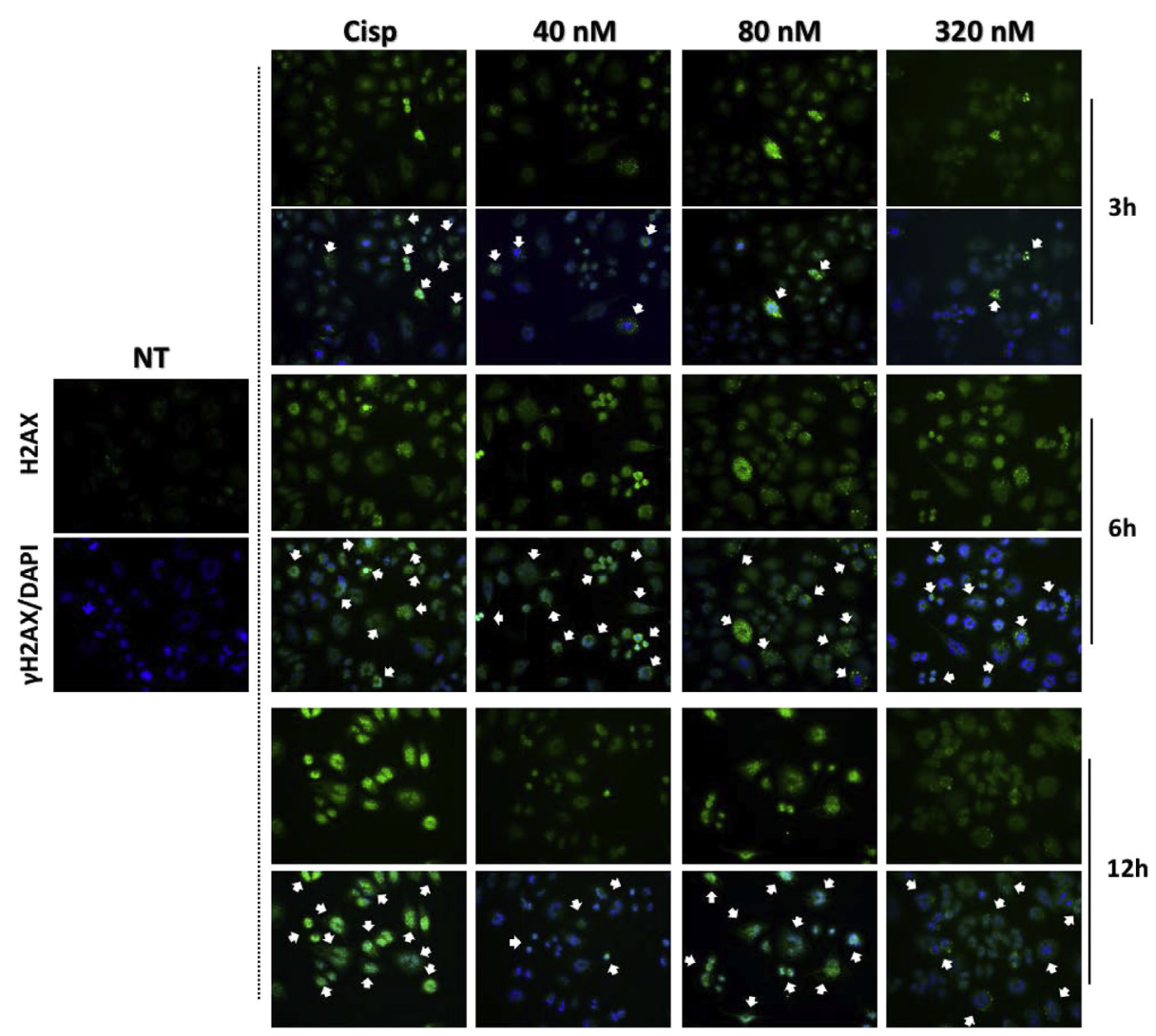

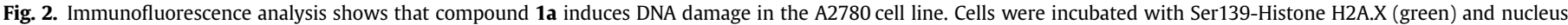

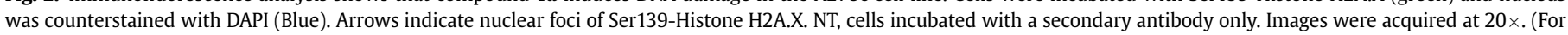
interpretation of the references to colour in this figure legend, the reader is referred to the Web version of this article.) 

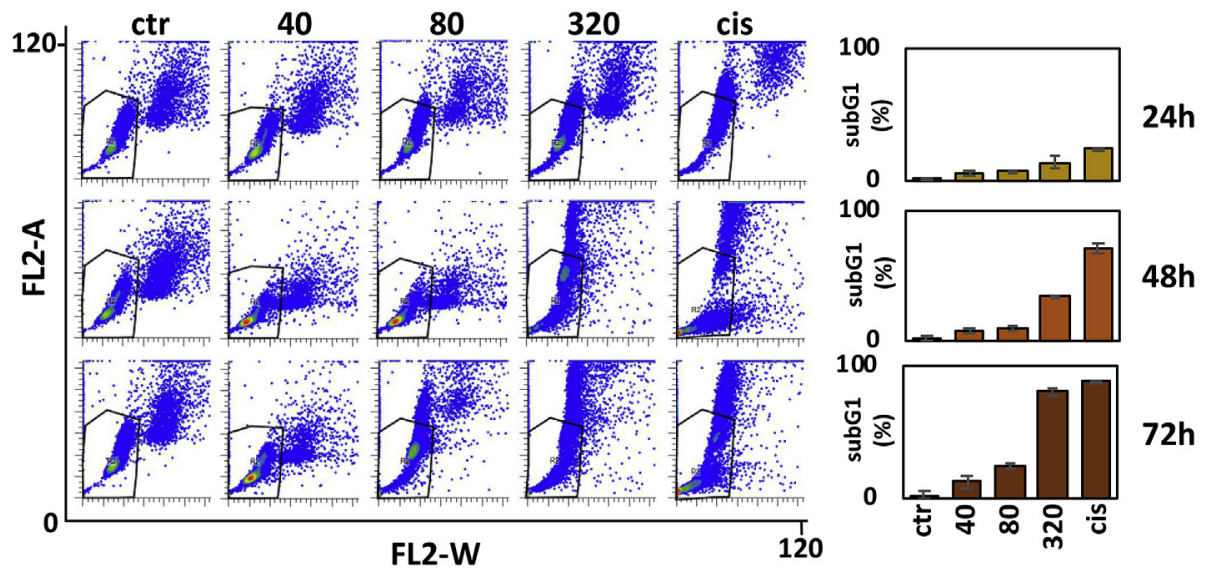

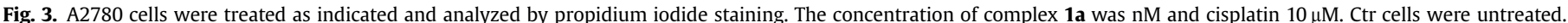
Histograms showed the average and standard deviation of two independent experiments. Y axis indicates the percentage of cells in sub G1.

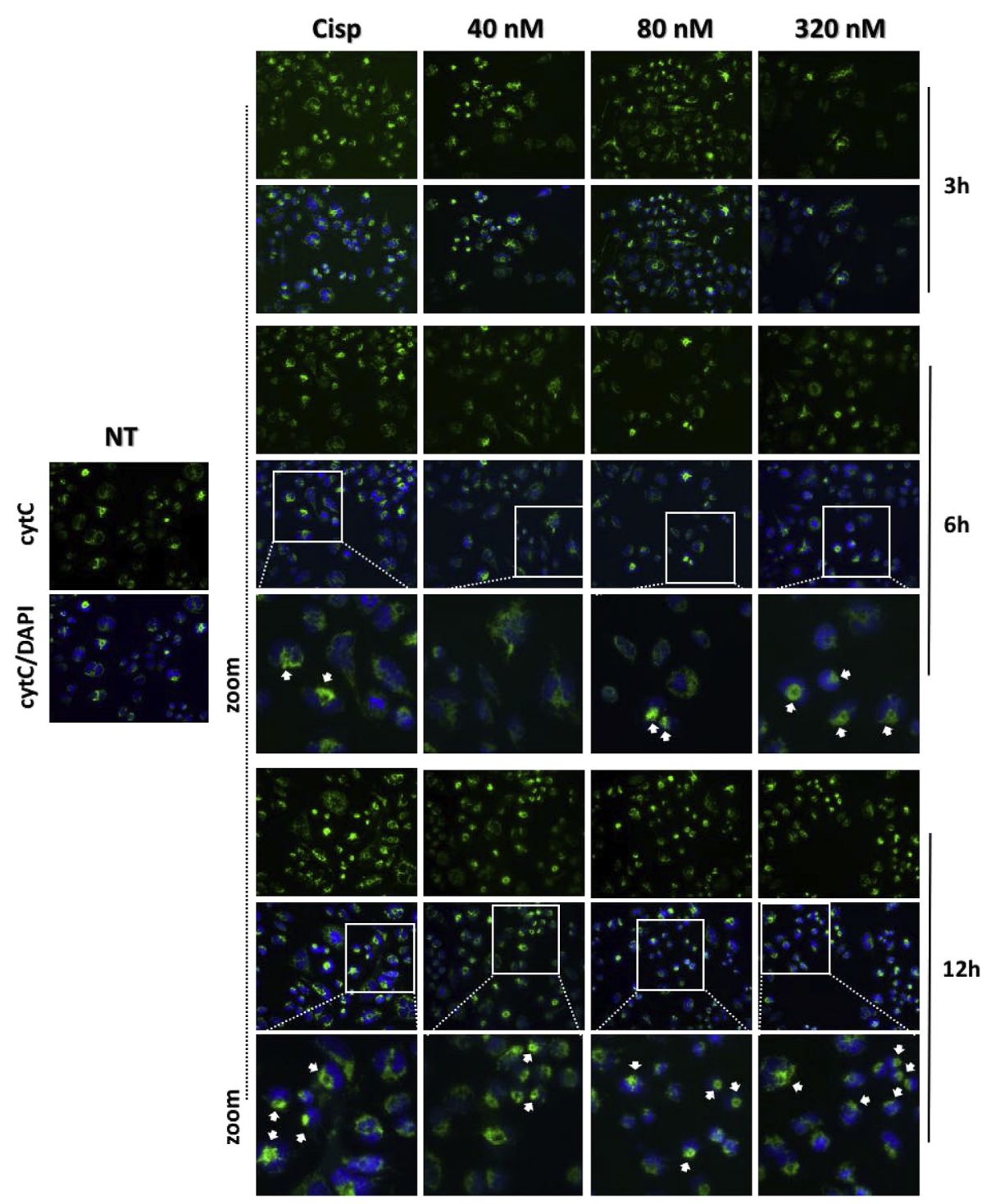

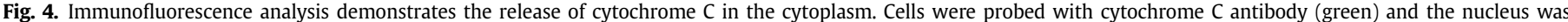

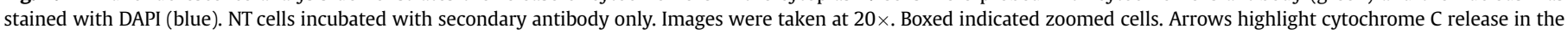
cytoplasm. (For interpretation of the references to colour in this figure legend, the reader is referred to the Web version of this article.) 


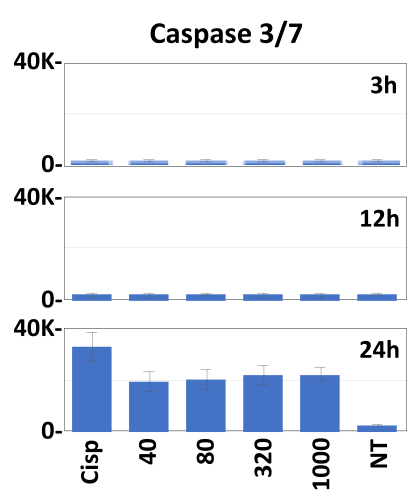

Fig. 5. A2780 cells were treated as indicated and the activity of caspase $3 / 7$ was measured. Complex 1a induced caspase 3/7 activation starting from low concentrations (40 nM).

complexes with chelating bis-N-heterocyclic carbene ligands. The anti-proliferative activities of these new compounds and those of other palladacyclopentadienyl complexes with different supporting ligands, previously prepared by our group, were systematically tested on different human tumor cell lines. The analysis of the results has shown that the combination of a palladacyclopentadienyl organometallic function with at least one NHC ligand assures a good cytotoxicity, sometimes significantly higher than cisplatin, against many cancer cell lines. Moreover, these derivatives are almost inactive toward normal MRC- 5 cells. One of the most intriguing results concerns the bis-NHC derivative 1a, which exhibits a particularly high activity against all ovarian cancer cell lines, matching (or improving) the results of the best palladium complexes reported in the literature $[7 c, e]$. Furthermore, this complex is stable in the presence of an excess of GSH and could reach unaltered its potential bio-target, thereby behaving as an authentic structural compound.

Platinum based therapy is the first line treatment in high grade serous ovarian cancer. Unfortunately, almost all patients relapse with a 5-year survival rate of less than $30 \%$. Here, we show that compound 1a acts primarily on DNA similarly to the platinum agent, representing a valid alternative to current therapy. Further experiments are required to understand the exact mechanism of action but a new hope for patient is on the way.

\section{Experimental}

All syntheses of complexes were carried out using standard Schlenk techniques under an atmosphere of dry argon. Dichloromethane was firstly treated with $3 \AA$ molecular sieves and then distilled over $\mathrm{P}_{2} \mathrm{O}_{5}$. All the other chemicals were commercially available grade products and were used as purchased.

Complexes Aga-b [18] and the palladium precursor $\left[\mathrm{PdC}_{4}(\mathrm{COOMe})_{4}\right]_{n}[19]$ were synthesized according to published procedures.

The IR, ${ }^{1} \mathrm{H}$ and ${ }^{13} \mathrm{C}\left\{\mathrm{H}^{1}\right\}$-NMR spectra were recorded on a PerkinElmer Spectrum One spectrophotometer and on Bruker 300 or 400 Avance spectrometers, respectively.

Elemental analysis was carried out using an Elemental $\mathrm{CHN}$ "CUBO Micro Vario" analyzer.

\subsection{Synthesis of (1,2,3,4-tetrakis(carbomethoxy)-1,3-pentadienyl) palladium(II)(1,1'-dibenzyl-3,3'-methylenediimidazolin-2,2' - diylidene) (1a)}

$0.0391 \mathrm{~g}(0.100 \mathrm{mmol})$ of the precursor $\left[\mathrm{PdC}_{4}(\mathrm{COOMe})_{4}\right]_{\mathrm{n}}$ was dissolved in ca. $10 \mathrm{~mL}$ of anhydrous $\mathrm{CH}_{2} \mathrm{Cl}_{2}$ into a $50 \mathrm{~mL}$ two necked flask under inert atmosphere ( $\mathrm{Ar})$. The resulting mixture was treated with $0.0705 \mathrm{~g}(0.100 \mathrm{mmol})$ of Aga, previously dissolved in ca. $15 \mathrm{~mL}$ of anhydrous $\mathrm{CH}_{2} \mathrm{Cl}_{2}$, and stirred at RT for ca. $1 \mathrm{~h}$.

The precipitated $\mathrm{AgBr}$ was removed by filtration on a millipore membrane filter.

Addition of diethylether to the concentrated solution yielded the complex 1a as a brownish precipitate, which was filtered off on a gooch and washed with $n$-pentane.

$0.0654 \mathrm{~g}$ of 1a was obtained (yield 90\%).
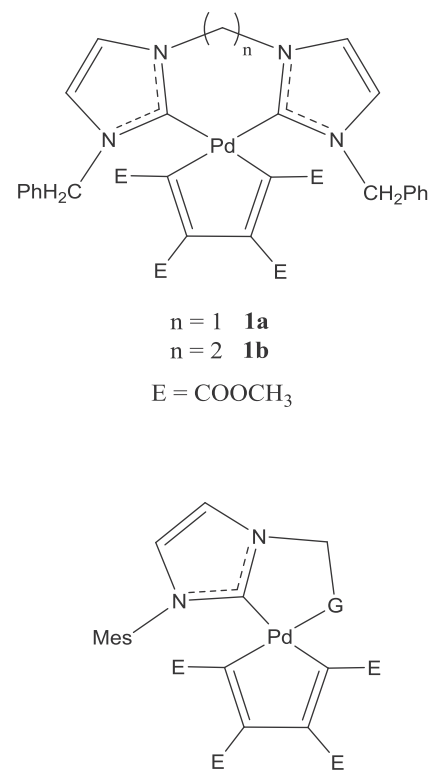

$\mathrm{G}=\mathrm{SCH}_{3} \mathbf{4 a}$

$\mathrm{G}=\mathrm{Py} \quad 4 \mathrm{~b}$

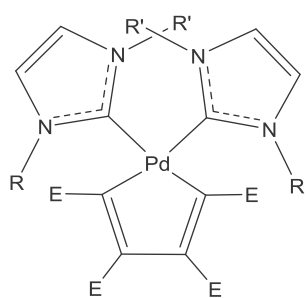

$\mathrm{R}=\mathrm{CH}_{3} \mathrm{R}^{\prime}=\mathrm{CH}_{2} \mathrm{Ph} \quad \mathbf{2 a}$ $\mathrm{R}=\mathrm{CH}_{3} \mathrm{R}^{\prime}=\mathrm{CH}_{2} \mathrm{Py} \quad \mathbf{2 b}$ $\mathrm{R}=\mathrm{CH}_{3} \mathrm{R}^{\prime}=\mathrm{CH}_{2} \mathrm{SCH}_{3} 2 \mathrm{c}$

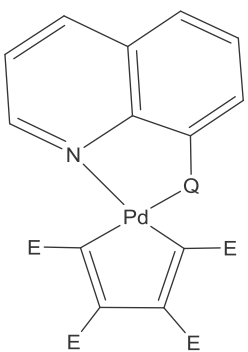

$\mathrm{Q}=\mathrm{PPh}_{2}$ 5a

$\mathrm{Q}=\mathrm{SCH}_{3} \mathbf{5 b}$

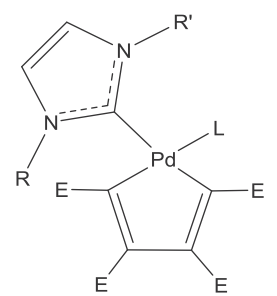

$\mathrm{L}=\mathrm{PPh}_{3} \quad \mathrm{R}=\mathrm{CH}_{3} \quad \mathrm{R}^{\prime}=\mathrm{CH}_{2}$ Tol 3a

$\mathrm{L}=\mathrm{PPh}_{3} \quad \mathrm{R}=\mathrm{CH}_{3} \quad \mathrm{R}^{\prime}=\mathrm{CH}_{2} \mathrm{Py} \quad \mathbf{3 b}$

$\mathrm{L}=\mathrm{DIC} \quad \mathrm{R}=\mathrm{CH}_{3} \quad \mathrm{R}^{\prime}=\mathrm{CH}_{2}$ Tol $3 \mathbf{c}$

$\mathrm{L}=\mathrm{DIC} \quad \mathrm{R}=\mathrm{CH}_{3} \mathrm{R}^{\prime}=\mathrm{CH}_{2} \mathrm{Py} \quad \mathbf{3 d}$

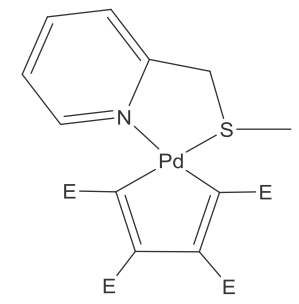

Chart 1. Palladacyclopentadienyl complexes studied in this work. 
${ }^{1} \mathrm{H}$ NMR $\left(400 \mathrm{MHz}, \mathrm{CDCl}_{3}, \mathrm{~T}=298 \mathrm{~K}, \mathrm{ppm}\right) \delta: 3.44(\mathrm{~s}, 6 \mathrm{H}, 2$ $\left.\mathrm{OCH}_{3}\right), 3.69\left(\mathrm{~s}, 6 \mathrm{H}, 2 \mathrm{OCH}_{3}\right), 4.98$ and 5.60 (AB system, $4 \mathrm{H}$, $\left.J=15.0 \mathrm{~Hz}, 2-\mathrm{CH}_{2} \mathrm{Ph}\right), 6.00$ and 6.49 (AB system, $2 \mathrm{H}, J=13.6 \mathrm{~Hz}$, $\left.\mathrm{NCH}_{2} \mathrm{~N}\right), 6.65\left(\overline{\mathrm{d}, 2} \mathrm{H}, J=1.8 \mathrm{~Hz}, 2 \mathrm{CH}^{\mathrm{Im}}\right), 6.99-7.26(10 \mathrm{H}, \mathrm{Ar}-\mathrm{H}), 7.32$ $\left(\mathrm{d}, 2 \mathrm{H}, J=1.8 \mathrm{~Hz}, 2 \mathrm{CH}^{\mathrm{Im}}\right)$.

${ }^{13} \mathrm{C}\left\{{ }^{1} \mathrm{H}\right.$ NMR $\left(\mathrm{CDCl}_{3}, \mathrm{~T}=298 \mathrm{~K}, \mathrm{ppm}\right) \delta: 50.7\left(\mathrm{CH}_{3}, \mathrm{OCH}_{3}\right), 51.3$ $\left(\mathrm{CH}_{3}, \mathrm{OCH}_{3}\right), 54.5\left(\mathrm{CH}_{2}, \mathrm{CH}_{2} \mathrm{Ph}\right), 65.5\left(\mathrm{CH}_{2}, \mathrm{NCH}_{2} \mathrm{~N}\right), 119.4(\mathrm{CH}$, $\left.\mathrm{CH}^{\mathrm{Im}}\right), 122.6\left(\mathrm{CH}, \mathrm{CH}^{\mathrm{Im}}\right), 127.8-136.4(\mathrm{Ar}-\mathrm{C}), 145.7\left(\mathrm{C}, \underline{\mathrm{C}}-\mathrm{COOCH}_{3}\right)$, $166.5\left(\mathrm{C}, \mathrm{COOCH}_{3}\right), 169.7\left(\mathrm{C}, \underline{\mathrm{C}}-\mathrm{COOCH}_{3}\right), 177.5\left(\mathrm{C}, \underline{\mathrm{COO}} \mathrm{CH}_{3}\right), 179.7$ (C, carbene).

IR (KBr): $v_{\mathrm{C}}={ }_{\mathrm{O}}=1733,1686, v_{\mathrm{C}-\mathrm{O}}=1194 \mathrm{~cm}^{-1}, 1159 \mathrm{~cm}^{-1}$.

Anal. Calcd. for $\mathrm{C}_{33} \mathrm{H}_{32} \mathrm{~N}_{4} \mathrm{O}_{8} \mathrm{Pd}$ : C 55.12, $\mathrm{H} 4.49$, N 7.79. Found: C 55.43, H 4.30, N 7.88.

4.2. Synthesis of (1,2,3,4-tetrakis(carbomethoxy)-1,3-pentadienyl) palladium(II)(1,1'-dibenzyl-3,3'-ethylenediimidazolin-2,2'diylidene) (1b)

Complex 1b was prepared in an analogous manner to that described for 1a starting from $0.0385 \mathrm{~g}$ of $\left[\mathrm{PdC}_{4}(\mathrm{COOMe})_{4}\right]_{\mathrm{n}}$ and $0.0707 \mathrm{~g}$ of Agb.

$0.0702 \mathrm{~g}$ (yield $97 \%$ ) of $\mathbf{1 b}$ was obtained.

${ }^{1} \mathrm{H}$ NMR $\left(400 \mathrm{MHz}, \mathrm{CDCl}_{3}, \mathrm{~T}=298 \mathrm{~K}, \mathrm{ppm}\right) \delta: 3.32(\mathrm{~s}, 6 \mathrm{H}, 2$ $\left.\mathrm{OCH}_{3}\right), 3.58\left(\mathrm{~s}, 6 \mathrm{H}, 2 \mathrm{OCH}_{3}\right), 4.35\left(\mathrm{~m}, 2 \mathrm{H}, \mathrm{NCH}_{2} \mathrm{CH}_{2} \mathrm{~N}\right), 5.02$ and 5.07 (AB system, $4 \mathrm{H}, \mathrm{J}=15.4 \mathrm{~Hz}, 2 \mathrm{CH}_{2} \mathrm{Ph}$ ), $5.33\left(\mathrm{~m}, 2 \mathrm{H}, \mathrm{NCH}_{2} \mathrm{CH}_{2} \mathrm{~N}\right), 6.56$ $\left(\mathrm{d}, 2 \mathrm{H}, J=1.9 \mathrm{~Hz}, 2 \mathrm{CH}^{\mathrm{Im}}\right), 6.93\left(\mathrm{~d}, 2 \mathrm{H}, J=1.9 \mathrm{~Hz}, 2 \mathrm{CH}^{\mathrm{Im}}\right), 6.86$ and 7.10-7.15 (10H, $\mathrm{Ar}-\mathrm{H})$.

${ }^{13} \mathrm{C}\left\{{ }^{1} \mathrm{H}\right\} \mathrm{NMR}\left(\mathrm{CDCl}_{3}, \mathrm{~T}=298 \mathrm{~K}, \mathrm{ppm}\right) \delta: 48.2\left(\mathrm{CH}_{2}, \mathrm{NCH}_{2} \mathrm{CH}_{2} \mathrm{~N}\right)$, $50.7\left(\mathrm{CH}_{3}, \mathrm{OCH}_{3}\right), 51.1\left(\mathrm{CH}_{3}, \mathrm{OCH}_{3}\right), 54.4\left(\mathrm{CH}_{2}, \mathrm{CH}_{2} \mathrm{Ph}\right), 120.5(\mathrm{CH}$, $\left.\mathrm{CH}^{\mathrm{Im}}\right), 121.9\left(\mathrm{CH}, \mathrm{CH}^{\mathrm{Im}}\right), 128.0-136.2(\mathrm{Ar}-\mathrm{C}), 144.8\left(\mathrm{C}, \underline{\mathrm{C}}-\mathrm{COOCH}_{3}\right)$, $165.9\left(\mathrm{C}, \mathrm{COOCH}_{3}\right), 169.9\left(\mathrm{C}, \underline{\mathrm{C}}-\mathrm{COOCH}_{3}\right), 177.4\left(\mathrm{C}, \underline{\mathrm{COO}} \mathrm{CH}_{3}\right), 178.9$ (C, carbene).

IR (KBr): $v_{\mathrm{C}}=0=1691, v_{\mathrm{C}-\mathrm{O}}=1202 \mathrm{~cm}^{-1}$.

Anal. Calcd. for $\mathrm{C}_{34} \mathrm{H}_{34} \mathrm{~N}_{4} \mathrm{O}_{8} \mathrm{Pd}$ : C 55.71, H 4.68, N 7.64. Found: C 55.51, H 4.49, N 7.52.

4.3. Synthesis of (1,2,3,4-tetrakis(carbomethoxy)-1,3-pentadienyl) palladium(II)bis( $\kappa^{1}-C-([1-$ benzyl-3-methyl]imidazolyl-2-ene)) (2a)

Complex 2a was synthesized according to published protocol [15c] (see Supplementary Material).

${ }^{1} \mathrm{H}$ NMR $\left(\mathrm{CDCl}_{3}, \mathrm{~T}=298 \mathrm{~K}, \mathrm{ppm}\right)$ syn-isomer $\delta: 3.31(\mathrm{~s}, 3 \mathrm{H}$, $\left.\mathrm{OCH}_{3}\right), 3.65\left(\mathrm{~s}, 3 \mathrm{H}, \mathrm{OCH}_{3}\right), 3.95\left(\mathrm{~s}, 3 \mathrm{H}, \mathrm{NCH}_{3}\right), 5.28(\mathrm{~d}, J=15.1 \mathrm{~Hz}, 1 \mathrm{H}$, $\left.\mathrm{NCH}_{2}\right), 5.74\left(\mathrm{~d}, J=15.1 \mathrm{~Hz}, 1 \mathrm{H}, \mathrm{NCH}_{2}\right), 6.55(\mathrm{~d}, J=1.9 \mathrm{~Hz}, 1 \mathrm{H}, \mathrm{CH}=\mathrm{CH}$ $\operatorname{Im}), 6.87(\mathrm{~d}, J=1.9 \mathrm{~Hz}, 1 \mathrm{H}, \mathrm{CH}=\mathrm{CH} \mathrm{Im}), 6.88-6.90(\mathrm{~m}, 2 \mathrm{H}, \mathrm{Ph})$, 7.20-7.30 (m, 3H, Ph); anti-isomer $\delta: 3.29\left(\mathrm{~s}, 3 \mathrm{H}, \mathrm{OCH}_{3}\right), 3.55(\mathrm{~s}, 3 \mathrm{H}$, $\mathrm{NCH}_{3}$ ), 3.64 (s, 3H, OCH $), 5.19$ (d, $\left.J=15.8 \mathrm{~Hz}, 1 \mathrm{H}, \mathrm{NCH}_{2}\right), 5.74$ (d, $\left.J=15.8 \mathrm{~Hz}, 1 \mathrm{H}, \mathrm{NCH}_{2}\right), 6.71(\mathrm{~s}, 2 \mathrm{H}, \mathrm{CH}=\mathrm{CH} \mathrm{Im}), 6.88-6.90(\mathrm{~m}, 2 \mathrm{H}$, $\mathrm{Ph}), 7.20-7.30(\mathrm{~m}, 3 \mathrm{H}, \mathrm{Ph})$.

${ }^{13} \mathrm{C}\left\{{ }^{1} \mathrm{H}\right\}$ NMR $\left(\mathrm{CDCl}_{3}, \mathrm{~T}=298 \mathrm{~K}, \mathrm{ppm}\right)$ syn-isomer $\delta: 38.1\left(\mathrm{CH}_{3}\right.$, $\left.\mathrm{NCH}_{3}\right), 50.5\left(\mathrm{CH}_{3}, \mathrm{OCH}_{3}\right), 50.9\left(\mathrm{CH}_{3}, \mathrm{OCH}_{3}\right), 54.0\left(\mathrm{CH}_{2}, \mathrm{NCH}_{2}\right), 120.3$ $(\mathrm{CH}, \mathrm{CH}=\mathrm{CH} \mathrm{Im}), 121.9(\mathrm{CH}, \mathrm{CH}=\mathrm{CH} \mathrm{Im}), 128.0(\mathrm{CH}, \mathrm{Ph} p-\mathrm{C}), 128.1$ ( $\mathrm{CH}, \mathrm{Ph} o-\mathrm{C}), 128.7$ (CH, Ph m-C), 135.2 (C, Ph i-C), 143.9 (C, C=C), $165.2(\mathrm{C}, \mathrm{C}=\mathrm{O}), 169.0(\mathrm{C}, \mathrm{C}=\mathrm{C}), 176.9(\mathrm{C}, \mathrm{C}=\mathrm{O}), 181.8$ (C, carbene); anti-isomer $\delta$ : $37.2\left(\mathrm{CH}_{3}, \mathrm{NCH}_{3}\right), 50.5\left(\mathrm{CH}_{3}, \mathrm{OCH}_{3}\right), 50.9\left(\mathrm{CH}_{3}, \mathrm{OCH}_{3}\right)$, $54.0\left(\mathrm{CH}_{2}, \mathrm{NCH}_{2}\right), 120.4(\mathrm{CH}, \mathrm{CH}=\mathrm{CH} \mathrm{Im}), 122.5(\mathrm{CH}, \mathrm{CH}=\mathrm{CH} \mathrm{Im})$, 126.7 (CH, Ph o-C),127.9 (CH, Ph p-C), 128.7 ( CH, Ph m-C), 135.9 (C, Ph $i-\mathrm{C}), 143.7$ (C, C=C), $165.1(\mathrm{C}, \mathrm{C}=\mathrm{O}), 169.0$ (C, C=C), 176.9 (C, $\mathrm{C}=$ O), 182.0 (C, carbene). IR ( $\mathrm{KBr}): 1692 \mathrm{~cm}^{-1}$ ( $\left.v_{\mathrm{CO}}\right)$. Anal. Calcd for $\mathrm{C}_{34} \mathrm{H}_{36} \mathrm{~N}_{4} \mathrm{O}_{8} \mathrm{Pd}: \mathrm{C}, 55.55 ; \mathrm{H}, 4.94 ; \mathrm{N}, 7.62$. Found C, 55.64; H, 4.92; N, $7.56 \%$.
4.4. Synthesis of (1,2,3,4-tetrakis(carbomethoxy)-1,3-pentadienyl) palladium(II)bis $\left(\kappa^{1}-C-([1-(2-p y r i d y l) m e t h y l e n e-3-m e t h y l]\right.$ imidazolyl-2-ene)) (2b)

Complex 2b was synthesized according to published protocol [15c] (see Supplementary Material).

${ }^{1} \mathrm{H}$ NMR $\left(\mathrm{CDCl}_{3}, \mathrm{~T}=298 \mathrm{~K}, \mathrm{ppm}\right)$ : syn-isomer $\delta: 3.18(\mathrm{~s}, 3 \mathrm{H}$, $\left.\mathrm{OCH}_{3}\right), 3.65\left(\mathrm{~s}, 3 \mathrm{H}, \mathrm{OCH}_{3}\right), 3.88\left(\mathrm{~s}, 3 \mathrm{H}, \mathrm{NCH}_{3}\right), 5.27,5.32$ (AB system, $\left.J=16.1 \mathrm{~Hz}, 2 \mathrm{H}, \mathrm{NCH}_{2}\right), 6.86(\mathrm{~d}, J=1.9 \mathrm{~Hz}, 1 \mathrm{H}, \mathrm{CH}=\mathrm{CH} \mathrm{Im}), 6.90(\mathrm{~d}$, $J=1.9 \mathrm{~Hz}, 1 \mathrm{H}, \mathrm{CH}=\mathrm{CH} \mathrm{Im}$ ), 7.12 (ddd, $J=7.7,4.9,1.0 \mathrm{~Hz}, 1 \mathrm{H}, 5-\mathrm{Pyr}$ ), 7.24 (d, $J=7.7 \mathrm{~Hz}, 1 \mathrm{H}, 3-\mathrm{Pyr}), 7.56$ (td, $J=7.7,1.7 \mathrm{~Hz}, 1 \mathrm{H}, 4-\mathrm{Pyr}), 8.42$ (d, $J=4.9 \mathrm{~Hz}, 1 \mathrm{H}, 6-\mathrm{Pyr})$; anti-isomer $\delta: 3.25\left(\mathrm{~s}, 3 \mathrm{H}, \mathrm{OCH}_{3}\right), 3.58$ (s, $\left.3 \mathrm{H}, \mathrm{NCH}_{3}\right), 3.63\left(\mathrm{~s}, 3 \mathrm{H}, \mathrm{OCH}_{3}\right), 5.32$, (d J $\left.=16.1 \mathrm{~Hz}, 1 \mathrm{H}, \mathrm{NCH}_{2}\right), 5.71$, (d $\left.J=16.1 \mathrm{~Hz}, 1 \mathrm{H}, \mathrm{NCH}_{2}\right), 6.65(\mathrm{~d}, J=1.9 \mathrm{~Hz}, 1 \mathrm{H}, \mathrm{CH}=\mathrm{CH} \operatorname{Im}), 6.78(\mathrm{~d}$, $J=1.9 \mathrm{~Hz}, 1 \mathrm{H}, \mathrm{CH}=\mathrm{CH} \mathrm{Im}$ ), 7.07 (d, $J=7.7 \mathrm{~Hz}, 1 \mathrm{H}, 3-\mathrm{Pyr}), 7.18$ (ddd, $J=7.7,4.9,1.0 \mathrm{~Hz}, 1 \mathrm{H}, 5-\mathrm{Pyr}$ ), 7.54 (td, $J=7.7,1.7 \mathrm{~Hz}, 1 \mathrm{H}, 4-\mathrm{Pyr}$ ), 8.47 (d, $J=4.9 \mathrm{~Hz}, 1 \mathrm{H}, 6-\mathrm{Pyr}$ ).

${ }^{13} \mathrm{C}\left\{{ }^{1} \mathrm{H}\right\}$ NMR $\left(\mathrm{CDCl}_{3}, \mathrm{~T}=298 \mathrm{~K}, \mathrm{ppm}\right)$ syn-isomer $\delta: 38.0\left(\mathrm{CH}_{3}\right.$, $\left.\mathrm{NCH}_{3}\right), 50.5\left(\mathrm{CH}_{3}, \mathrm{OCH}_{3}\right), 50.9\left(\mathrm{CH}_{3}, \mathrm{OCH}_{3}\right), 55.6\left(\mathrm{CH}_{2}, \mathrm{NCH}_{2}\right), 121.0$ $(\mathrm{CH}, \mathrm{CH}=\mathrm{CH} \mathrm{Im}), 121.9(\mathrm{CH}, \mathrm{CH}=\mathrm{CH} \mathrm{Im}), 122.6(\mathrm{CH}, 5-\mathrm{Pyr}), 123.5$ (CH, 3-Pyr), 136.8 (CH, 4-Pyr), 144.2 (C, C=C), 149.2 (C, 6-Pyr), 155.0 (CH, 2-Pyr), 165.2 (C, C=O), 169.2 (C, $C=C), 176.9(C, C=0), 181.3$ (C, $\mathrm{NCN})$; anti-isomer $\delta$ : $37.4\left(\mathrm{CH}_{3}, \mathrm{NCH}_{3}\right), 50.5\left(\mathrm{CH}_{3}, \mathrm{OCH}_{3}\right), 50.9\left(\mathrm{CH}_{3}\right.$, $\left.\mathrm{OCH}_{3}\right), 55.6\left(\mathrm{CH}_{2}, \mathrm{NCH}_{2}\right), 120.6(\mathrm{CH}, \mathrm{CH}=\mathrm{CH} \mathrm{Im}), 122.0(\mathrm{CH}, 3-\mathrm{Pyr})$, $122.3(\mathrm{CH}, \mathrm{CH}=\mathrm{CH} \mathrm{Im}), 122.7$ (CH, 5-Pyr), 136.8 (CH, 4-Pyr), 143.9 (C, C=C), 149.2 (C, 6-Pyr), 155.7 ( CH, 2-Pyr), 165.3 (C, C=O), 169.2 $(\mathrm{C}, \mathrm{C}=\mathrm{C}), 176.9$ (C, $\mathrm{C}=\mathrm{O}), 182.0$ (C, carbene). IR $(\mathrm{KBr}): 1692 \mathrm{~cm}^{-1}$ $\left(v_{\text {CO }}\right)$. Anal. Calcd for $\mathrm{C}_{32} \mathrm{H}_{34} \mathrm{~N}_{6} \mathrm{O}_{8} \mathrm{Pd}$ : C, 52.14; H, 4.65; N, 11.40 . Found C, 52.20; $\mathrm{H}, 4.56 ; \mathrm{N}, 11.33 \%$.

\subsection{Synthesis of (1,2,3,4-tetrakis(carbomethoxy)-1,3-pentadienyl) palladium(II)bis( $\kappa^{1}-\mathrm{C}-([1-($ methylthio)methylene-3-methyl] imidazolyl-2-ene)) (2c)}

Complex 2c was synthesized according to published protocol [15c] (see Supplementary Material).

${ }^{1} \mathrm{H}$ NMR $\left(\mathrm{CDCl}_{3}, \mathrm{~T}=298 \mathrm{~K}, \mathrm{ppm}\right)$ : syn-isomer $\delta: 2.10(\mathrm{~s}, 3 \mathrm{H}$, $\left.\mathrm{SCH}_{3}\right), 3.31\left(\mathrm{~s}, 3 \mathrm{H}, \mathrm{OCH}_{3}\right), 3.63\left(\mathrm{~s}, 3 \mathrm{H}, \mathrm{OCH}_{3}\right), 3.83\left(\mathrm{~s}, 3 \mathrm{H}, \mathrm{NCH}_{3}\right), 4.94$ $\left(\mathrm{d}, J=13.8 \mathrm{~Hz}, 1 \mathrm{H}, \mathrm{SCH}_{2}\right), 5.09\left(\mathrm{~d}, J=13.9 \mathrm{~Hz}, 1 \mathrm{H}, \mathrm{SCH}_{2}\right), 6.92$ (d, $J=1.9 \mathrm{~Hz}, 1 \mathrm{H}, \mathrm{CH}=\mathrm{CH} \mathrm{Im}), 7.26(\mathrm{~d}, J=1.9 \mathrm{~Hz}, 1 \mathrm{H}, \mathrm{CH}=\mathrm{CH} \mathrm{Im})$; antiisomer $\delta$ : $1.87\left(\mathrm{~s}, 3 \mathrm{H}, \mathrm{SCH}_{3}\right), 3.30\left(\mathrm{~s}, 3 \mathrm{H}, \mathrm{OCH}_{3}\right), 3.63\left(\mathrm{~s}, 3 \mathrm{H}, \mathrm{OCH}_{3}\right)$, $4.00\left(\mathrm{~s}, 3 \mathrm{H}, \mathrm{NCH}_{3}\right), 4.94\left(\mathrm{~d}, J=13.9 \mathrm{~Hz}, 1 \mathrm{H}, \mathrm{SCH}_{2}\right), 5.26(\mathrm{~d}$, $\left.J=13.9 \mathrm{~Hz}, 1 \mathrm{H}, \mathrm{SCH}_{2}\right), 6.96(\mathrm{~d}, J=1.9 \mathrm{~Hz}, 1 \mathrm{H}, \mathrm{CH}=\mathrm{CH} \mathrm{Im}), 7.19$ (d, $J=1.9 \mathrm{~Hz}, 1 \mathrm{H}, \mathrm{CH}=\mathrm{CH} \mathrm{Im})$.

${ }^{13} \mathrm{C}\left\{{ }^{1} \mathrm{H}\right\}$ NMR $\left(\mathrm{CDCl}_{3}, \mathrm{~T}=298 \mathrm{~K}, \mathrm{ppm}\right)$ syn-isomer $\delta: 15.2\left(\mathrm{CH}_{3}\right.$, $\left.\mathrm{SCH}_{3}\right), 38.1\left(\mathrm{CH}_{3}, \mathrm{NCH}_{3}\right), 50.5\left(\mathrm{CH}_{3}, \mathrm{OCH}_{3}\right), 50.9\left(\mathrm{CH}_{3}, \mathrm{OCH}_{3}\right), 53.3$ $\left(\mathrm{CH}_{2}, \mathrm{SCH}_{2}\right), 119.9(\mathrm{CH}, \mathrm{CH}=\mathrm{CH} \mathrm{Im}), 122.4(\mathrm{CH}, \mathrm{CH}=\mathrm{CH} \mathrm{Im}), 144.3$ (C, $C=C), 165.1(C, C=0), 168.5(C, C=C), 176.7(C, C=0), 181.3$ (C, carbene). anti-isomer $\delta$ : $13.9\left(\mathrm{CH}_{3}, \mathrm{SCH}_{3}\right), 38.3\left(\mathrm{CH}_{3}, \mathrm{NCH}_{3}\right), 50.5$ $\left(\mathrm{CH}_{3}, \mathrm{OCH}_{3}\right), 50.9\left(\mathrm{CH}_{3}, \mathrm{OCH}_{3}\right), 54.0\left(\mathrm{CH}_{2}, \mathrm{SCH}_{2}\right), 119.7(\mathrm{CH}, \mathrm{CH}=\mathrm{CH}$ Im), $122.7(\mathrm{CH}, \mathrm{CH}=\mathrm{CH} \mathrm{Im}), 144.3(\mathrm{C}, \mathrm{C}=\mathrm{C}), 165.1(\mathrm{C}, \mathrm{C}=\mathrm{O}), 168.4$ $(\mathrm{C}, \mathrm{C}=\mathrm{C}), 176.7(\mathrm{C}, \mathrm{C}=\mathrm{O}), 181.5$ (C, carbene). IR $(\mathrm{KBr}): 1692 \mathrm{~cm}^{-1}$ $\left(v_{\mathrm{CO}}\right)$. Anal. Calcd for $\mathrm{C}_{24} \mathrm{H}_{32} \mathrm{~N}_{4} \mathrm{O}_{8} \mathrm{PdS}_{2}$ : C, 42.70; H, 4.78; N, 8.30. Found C, 42.88; $\mathrm{H}, 4.88 ; \mathrm{N}, 8.39 \%$.

4.6. Synthesis of (1,2,3,4-tetrakis(carbomethoxy)-1,3-pentadienyl) palladium(II)( $\kappa^{1}-C-([1-(4-m e t h y l p h e n y l) m e t h y l e n e-3-m e t h y l]$ imidazolyl-2-ene))(triphenylphosphine) (3a)

Complex 3a was synthesized according to published protocol [15c] (see Supplementary Material).

${ }^{1} \mathrm{H}$ NMR $\left(\mathrm{CDCl}_{3}, \mathrm{~T}=298 \mathrm{~K}, \mathrm{ppm}\right) \delta: 2.33\left(\mathrm{~s}, 3 \mathrm{H}\right.$, tolyl- $\left.\mathrm{CH}_{3}\right), 2.56$ $\left(\mathrm{s}, 3 \mathrm{H}, \mathrm{OCH}_{3}\right), 3.21\left(\mathrm{~s}, 3 \mathrm{H}, \mathrm{OCH}_{3}\right), 3.33\left(\mathrm{~s}, 3 \mathrm{H}, \mathrm{NCH}_{3}\right), 3.62(\mathrm{~s}, 3 \mathrm{H}$, $\left.\mathrm{OCH}_{3}\right), 3.65\left(\mathrm{~s}, 3 \mathrm{H}, \mathrm{OCH}_{3}\right), 4.56\left(\mathrm{~d}, J=13.8 \mathrm{~Hz}, 1 \mathrm{H}, \mathrm{N}-\mathrm{CH}_{2}\right), 5.22(\mathrm{~d}$, $\left.J=13.8 \mathrm{~Hz}, 1 \mathrm{H}, \mathrm{N}-\mathrm{CH}_{2}\right), 6.29(\mathrm{~d}, J=1.8 \mathrm{~Hz}, 1 \mathrm{H}, \mathrm{CH}=\mathrm{CH} \mathrm{Im}), 6.51(\mathrm{~d}$, 
$J=1.8 \mathrm{~Hz}, 1 \mathrm{H}, \mathrm{CH}=\mathrm{CH} \operatorname{Im}), 6.86(\mathrm{~d}, J=7.9 \mathrm{~Hz}, 2 \mathrm{H}$, tolyl $m-\mathrm{H}), 7.06(\mathrm{~d}$, $J=7.9 \mathrm{~Hz}, 2 \mathrm{H}$, tolyl $o-\mathrm{H}), 7,29-7.44\left(\mathrm{~m}, 15 \mathrm{H}, \mathrm{PPh}_{3}\right)$.

${ }^{13} \mathrm{C}\left\{{ }^{1} \mathrm{H}\right\}$ NMR $\left(\mathrm{CDCl}_{3}, \mathrm{~T}=298 \mathrm{~K}, \mathrm{ppm}\right) \delta: 21.1\left(\mathrm{CH}_{3}\right.$, tolyl- $\left.\mathrm{CH}_{3}\right)$, $37.0\left(\mathrm{CH}_{3}, \mathrm{NCH}_{3}\right), 49.8\left(\mathrm{CH}_{3}, \mathrm{OCH}_{3}\right), 50.7\left(\mathrm{CH}_{3}, \mathrm{OCH}_{3}\right), 51.0\left(\mathrm{CH}_{3}\right.$, $\left.\mathrm{OCH}_{3}\right), 51.1\left(\mathrm{CH}_{3}, \mathrm{OCH}_{3}\right), 53.8\left(\mathrm{CH}_{2}, \mathrm{NCH}_{2}\right), 120.0(\mathrm{CH}, \mathrm{CH}=\mathrm{CH} \mathrm{Im})$, $121.5(\mathrm{CH}, \mathrm{CH}=\mathrm{CH} \mathrm{Im}), 129.4(\mathrm{CH}$, tolyl $o-\mathrm{C}), 129.9(\mathrm{CH}$, tolyl $m-\mathrm{C})$, 130.6 (C, tolyl $i-C), 138.3(\mathrm{C}$, tolyl $p-\mathrm{C}), 145.5\left(\mathrm{C}, \mathrm{d}, \mathrm{J}_{\mathrm{CP}}=5.7 \mathrm{~Hz}, \mathrm{C}=\mathrm{C}\right)$, $148.2\left(\mathrm{C}, \mathrm{d}, \mathrm{J}_{\mathrm{CP}}=7.5 \mathrm{~Hz}, \mathrm{C}=\mathrm{C}\right), 165.1\left(\mathrm{C}, \mathrm{d}, \mathrm{J}_{\mathrm{CP}}=9.7 \mathrm{~Hz}, \mathrm{CO}\right), 166.6$ (C,CO), 170.0 (C, C = C), $171.5(\mathrm{C}, \mathrm{C}=\mathrm{C}), 174.9$ (C, d, J $\mathrm{CP}=4.6 \mathrm{~Hz}, \mathrm{CO})$, 175.7 (C, d, J JP $=5.1 \mathrm{~Hz}, \mathrm{CO}), 178.9\left(\mathrm{C}, \mathrm{d}, \mathrm{J}_{\mathrm{CP}}=16.5 \mathrm{~Hz}\right.$, carbene). ${ }^{31} \mathrm{P}$ $\left\{{ }^{1} \mathrm{H}\right\} \mathrm{NMR}\left(\mathrm{CDCl}_{3}, \mathrm{~T}=298 \mathrm{~K}, \mathrm{ppm}\right) \delta: 27.5 . \mathrm{IR}(\mathrm{KBr}): \nu_{\mathrm{C}}=\mathrm{O}=1695$, $1794 \mathrm{~cm}^{-11}\left(v_{\mathrm{CO}}\right)$. Anal. Calcd for $\mathrm{C}_{42} \mathrm{H}_{41} \mathrm{~N}_{2} \mathrm{O}_{8} \mathrm{PPd}$ : C, 60.11; H, 4.92; $\mathrm{N}, 3.34$. Found C, 60.27; $\mathrm{H}, 4.84 ; \mathrm{N}, 3.39 \%$.

4.7. Synthesis of (1,2,3,4-tetrakis(carbomethoxy)-1,3-pentadienyl) palladium(II)( $\kappa^{1}-\mathrm{C}-([1-(2-p y r i d y l) m e t h y l e n e-3-m e t h y l] i m i d a z o l y l-$ 2-ene))(triphenylphosphine) (3b)

Complex 3b was synthesized according to published protocol [15c] (see Supplementary Material).

${ }^{1} \mathrm{H}$ NMR $\left(\mathrm{CDCl}_{3}, \mathrm{~T}=298 \mathrm{~K}, \mathrm{ppm}\right) \delta: 2.55\left(\mathrm{~s}, 3 \mathrm{H}, \mathrm{OCH}_{3}\right), 3.10(\mathrm{~s}, 3 \mathrm{H}$, $\left.\mathrm{OCH}_{3}\right), 3.23\left(\mathrm{~s}, 3 \mathrm{H}, \mathrm{NCH}_{3}\right), 3.63(\mathrm{~s}, 3 \mathrm{H}, \mathrm{OCH} 3), 3.64\left(\mathrm{~s}, 3 \mathrm{H}, \mathrm{OCH}_{3}\right)$, $5.08\left(\mathrm{~d}, J=14.6 \mathrm{~Hz}, 1 \mathrm{H}, \mathrm{NCH}_{2}\right), 5.32\left(\mathrm{~d}, J=14.8 \mathrm{~Hz}, 1 \mathrm{H}, \mathrm{NCH}_{2}\right), 6.50$ $(\mathrm{d}, J=1.8 \mathrm{~Hz}, 1 \mathrm{H}, \mathrm{CH}=\mathrm{CH} \mathrm{Im}), 6.95(\mathrm{~d}, J=1.8 \mathrm{~Hz}, 1 \mathrm{H}, \mathrm{CH}=\mathrm{CH} \mathrm{Im})$, 7.14 (m, 1H, 5-Pyr), 7.22-7.39 (m, 16H, 3-Pyr, PPh), 7.52 (td, J=7.5, $1.6 \mathrm{~Hz}, 1 \mathrm{H}, 4-\mathrm{Pyr}$ ), 8.28 (d, $J=4.7 \mathrm{~Hz}, 1 \mathrm{H}, 6-\mathrm{Pyr}$ ).

${ }^{13} \mathrm{C}\left\{{ }^{1} \mathrm{H}\right\}$ NMR $\left(\mathrm{CDCl}_{3}, \mathrm{~T}=298 \mathrm{~K}, \mathrm{ppm}\right) \delta: 36.8\left(\mathrm{CH}_{3}, \mathrm{NCH}_{3}\right), 49.8$ $\left(\mathrm{CH}_{3}, \mathrm{OCH}_{3}\right), 50.6\left(\mathrm{CH}_{3}, \mathrm{OCH}_{3}\right), 51.0\left(\mathrm{CH}_{3}, \mathrm{OCH}_{3}\right), 51.1\left(\mathrm{CH}_{3}, \mathrm{OCH}_{3}\right)$, $55.4\left(\mathrm{CH}_{2}, \mathrm{NCH}_{2}\right), 121.5(\mathrm{CH}, \mathrm{CH}=\mathrm{CH} \mathrm{Im}), 122.0(\mathrm{CH}, \mathrm{CH}=\mathrm{CH} \mathrm{Im})$, 122.8 (CH, 5-Pyr), 124.2 (CH, 3-Pyr), 136.8 (CH, 4-Pyr), 145.2 (C, C= C), 148.1 (C, C=C), 149.0 (C, 6-Pyr), 154.1 ( CH, 2-Pyr), 164.3 (C, C= O), 165.1 ( $C, C=C), 166.5$ (C, $C=0), 174.9$ ( $C, C=C), 175.0$ ( $C, C=0$ ), 175.7 (C, $C=0), 179.2$ (C, carbene). ${ }^{31} \mathrm{P}\left\{{ }^{1} \mathrm{H}\right\}$ NMR $\left(\mathrm{CDCl}_{3}, \mathrm{~T}=298 \mathrm{~K}\right.$, ppm) $\delta: 27.4\left(\mathrm{~s}, \mathrm{PPh}_{3}\right)$. IR $(\mathrm{KBr}): 1697 \mathrm{~cm}^{-1}\left(v_{\mathrm{CO}}\right)$. Anal. Calcd for $\mathrm{C}_{40} \mathrm{H}_{38} \mathrm{~N}_{3} \mathrm{O}_{8} \mathrm{PPd}$ : C, 58.15; H, 4.64; N, 5.09. Found C, 58.20; H, 4.70; $\mathrm{N}, 5.18 \%$.

4.8. Synthesis of (1,2,3,4-tetrakis(carbomethoxy)-1,3-pentadienyl) palladium(II)( $\kappa^{1}-\mathrm{C}-([1-(4-$ methylphenyl)methylene-3-methyl] imidazolyl-2-ene))(2-isocyano-1,3-dimethylbenzene) (3c)

Complex 3c was synthesized according to published protocol [15c] (see Supplementary Material).

${ }^{1} \mathrm{H} \operatorname{NMR}\left(\mathrm{CDCl}_{3}, \mathrm{~T}=298 \mathrm{~K}, \mathrm{ppm}\right) \delta: 2.13\left(\mathrm{~s}, 6 \mathrm{H}\right.$, aryl- $\left.\mathrm{CH}_{3}\right), 2.15(\mathrm{~s}$, $3 \mathrm{H}$, tol- $\left.\mathrm{CH}_{3}\right), 3.31\left(\mathrm{~s}, 3 \mathrm{H}, \mathrm{OCH}_{3}\right), 3.59\left(\mathrm{~s}, 3 \mathrm{H}, \mathrm{OCH}_{3}\right), 3.65(\mathrm{~s}, 3 \mathrm{H}$, $\left.\mathrm{OCH}_{3}\right), 3.68\left(\mathrm{~s}, 3 \mathrm{H}, \mathrm{OCH}_{3}\right), 3.87\left(\mathrm{~s}, 3 \mathrm{H}, \mathrm{NCH}_{3}\right), 5.09(\mathrm{~d}, J=15.0 \mathrm{~Hz}, 1 \mathrm{H}$, $\left.\mathrm{N}-\mathrm{CH}_{2}\right), 5.58\left(\mathrm{~d}, J=15.0 \mathrm{~Hz}, 1 \mathrm{H}, \mathrm{N}-\mathrm{CH}_{2}\right), 6.94-6.97(\mathrm{~m}, 4 \mathrm{H}, \mathrm{CH}=$ $\mathrm{CH}$ Im, tol $m-\mathrm{H}), 7.06(\mathrm{~d}, J=7.6 \mathrm{~Hz}, 2 \mathrm{H}$, aryl $m-\mathrm{H}), 7.14(\mathrm{~d}, J=8.0 \mathrm{~Hz}$, $2 \mathrm{H}$, tol $o-\mathrm{H}), 7.21(\mathrm{t}, J=7.6 \mathrm{~Hz}$, aryl $p-\mathrm{H}) .{ }^{13} \mathrm{C}\left\{{ }^{1} \mathrm{H}\right\}$ NMR $\left(\mathrm{CDCl}_{3}\right.$, $\mathrm{T}=298 \mathrm{~K}, \mathrm{ppm}) \delta: 18.6\left(\mathrm{CH}_{3}\right.$, aryl- $\left.\mathrm{CH}_{3}\right), 21.3\left(\mathrm{CH}_{3}\right.$, tol- $\left.\mathrm{CH}_{3}\right), 38.2$ $\left(\mathrm{CH}_{3}, \mathrm{NCH}_{3}\right), 51.2\left(\mathrm{CH}_{3}, \mathrm{OCH}_{3}\right), 51.3\left(\mathrm{CH}_{3}, \mathrm{OCH}_{3}\right), 51.5\left(\mathrm{CH}_{3}, \mathrm{OCH}_{3}\right)$, 51.6 $\left(\mathrm{CH}_{3}, \mathrm{OCH}_{3}\right), 54.8\left(\mathrm{CH}_{2}, \mathrm{NCH}_{2}\right), 121.9(\mathrm{CH}, \mathrm{CH}=\mathrm{CH} \mathrm{Im}), 122.8$ $(\mathrm{CH}, \mathrm{CH}=\mathrm{CH} \mathrm{Im}), 126.5(\mathrm{C}$, aryl $i-\mathrm{C}), 128.3(\mathrm{CH}$, aryl $m-\mathrm{C}), 128.4(\mathrm{CH}$, tol o-C), $129.7(\mathrm{CH}$, tol $m-\mathrm{C}), 129.9(\mathrm{CH}$, aryl $p-\mathrm{C}), 133.3$ (C, tol $i-\mathrm{C})$, 135.5 (C, aryl o-C), $138.3(\mathrm{C}$, tol $p-\mathrm{C}), 145.9(\mathrm{C}, \mathrm{C}=\mathrm{C}), 146.7(\mathrm{C}, \mathrm{C}=\mathrm{C})$, 150.5 (C,CN), 164.8 (C, CO), 165.6 (C,CO), 166.6 (C, C =C), 166.9 (C, $\mathrm{C}=\mathrm{C}), 175.7$ (C, CO), 176.5 (C, CO), 178.4 (C, carbene). IR ( $\mathrm{KBr})$ : $v_{\mathrm{CO}}=1697 ; v_{\mathrm{CN}}=2174 \mathrm{~cm}^{-1}$. Anal. Calcd for $\mathrm{C}_{33} \mathrm{H}_{35} \mathrm{~N}_{3} \mathrm{O}_{8} \mathrm{Pd}$ : C, 55.98; H, 4.98; N, 5.93. Found C, 55.88; H, 4.88; N, 5.98\%.

4.9. Synthesis of (1,2,3,4-tetrakis(carbomethoxy)-1,3-pentadienyl) palladium $(I I)\left(\kappa^{1}-C-([1-(2-p y r i d y l) m e t h y l e n e-3-m e t h y l] i m i d a z o l y l-\right.$ 2-ene)(2-isocyano-1,3-dimethylbenzene) (3d)

Complex 3d was synthesized according to published protocol [15c] (see Supplementary Material).

${ }^{1} \mathrm{H} \mathrm{NMR}\left(\mathrm{CDCl}_{3}, \mathrm{~T}=298 \mathrm{~K}, \mathrm{ppm}\right) \delta: 2.10\left(\mathrm{~s}, 6 \mathrm{H}\right.$, aryl- $\left.\mathrm{CH}_{3}\right), 3.29(\mathrm{~s}$, $\left.3 \mathrm{H}, \mathrm{OCH}_{3}\right), 3.61\left(\mathrm{~s}, 3 \mathrm{H}, \mathrm{OCH}_{3}\right), 3.66\left(\mathrm{~s}, 3 \mathrm{H}, \mathrm{OCH}_{3}\right), 3.69\left(\mathrm{~s}, 3 \mathrm{H}, \mathrm{OCH}_{3}\right)$, $3.86\left(\mathrm{~s}, 3 \mathrm{H}, \mathrm{NCH}_{3}\right), 5.22\left(\mathrm{~d}, J=14.8 \mathrm{~Hz}, 1 \mathrm{H}, \mathrm{NCH}_{2}\right), 5.65(\mathrm{~d}$, $\left.J=14.8 \mathrm{~Hz}, 1 \mathrm{H}, \mathrm{NCH}_{2}\right), 6.97(\mathrm{~d}, J=1.8 \mathrm{~Hz}, 1 \mathrm{H}, \mathrm{CH}=\mathrm{CH} \mathrm{Im}), 7.01(\mathrm{~m}$ 1H, 5-Pyr,), 7.04 (d, J=7.8 Hz, 2H, aryl m-H), 7.20 (d, J=7.8 Hz, $1 \mathrm{H}$ aryl p-H), $7.21(\mathrm{~d}, J=1.8 \mathrm{~Hz}, 1 \mathrm{H}, \mathrm{CH}=\mathrm{CH} \mathrm{Im}), 7.60-7.63(\mathrm{~m}, 2 \mathrm{H}, 3-$ Pyr, 4-Pyr), 8.28 (d, $J=4.8 \mathrm{~Hz}, 1 \mathrm{H}, 6-\mathrm{Pyr}$ ).

${ }^{13} \mathrm{C}\left\{{ }^{1} \mathrm{H}\right\} \operatorname{NMR}\left(\mathrm{CDCl}_{3}, \mathrm{~T}=298 \mathrm{~K}, \mathrm{ppm}\right) \delta: 18.1\left(\mathrm{CH}_{3}, \mathrm{Ar}-\mathrm{CH}_{3}\right), 37.8$ $\left(\mathrm{CH}_{3}, \mathrm{NCH}_{3}\right), 50.7\left(\mathrm{CH}_{3}, \mathrm{OCH}_{3}\right), 50.8\left(\mathrm{CH}_{3}, \mathrm{OCH}_{3}\right), 51.1\left(\mathrm{CH}_{3}, \mathrm{OCH}_{3}\right)$, $51.2\left(\mathrm{CH}_{3}, \mathrm{OCH}_{3}\right), 55.9\left(\mathrm{CH}_{2}, \mathrm{NCH}_{2}\right), 122.0(\mathrm{CH}, \mathrm{CH}=\mathrm{CH} \mathrm{Im}), 122.5$ (CH, 5-Pyr), $122.8(\mathrm{CH}, \mathrm{CH}=\mathrm{CH} \mathrm{Im}), 123.9(\mathrm{CH}, 3-\mathrm{Pyr}), 126.7$ (C, aryl $\mathrm{i}-\mathrm{C}), 127.8(\mathrm{CH}$, aryl $\mathrm{m}-\mathrm{C}), 129.5(\mathrm{CH}$, aryl p-C), 135.1 (C, aryl o-C), 136.9 ( $\mathrm{CH}, 4-\mathrm{Pyr}), 145.3$ (C, C=C), 146.3 (C, C=C), 149.3 (C, 6-Pyr), $155.1(\mathrm{CH}, 2-\mathrm{Pyr}), 164.4(\mathrm{C}, \mathrm{C}=\mathrm{O}), 165.1(\mathrm{C}, \mathrm{C}=\mathrm{O}), 166.2(\mathrm{C}, \mathrm{C}=\mathrm{C})$, $166.3(C, C=C), 175.2(C, C=0), 176.1(C, C=0), 177.8$ (C, carbene). IR $(\mathrm{KBr}): 1697 \mathrm{~cm}^{-1}\left(v_{\mathrm{CO}}\right) ; 2174 \mathrm{~cm}^{-1}\left(v_{\mathrm{CN}}\right)$. Anal. Calcd for $\mathrm{C}_{31} \mathrm{H}_{32} \mathrm{~N}_{4} \mathrm{O}_{8} \mathrm{Pd}: \mathrm{C}, 53.57 ; \mathrm{H}, 4.64 ; \mathrm{N}, 8.06$. Found $\mathrm{C}, 53.64 ; \mathrm{H}, 4.56 ; \mathrm{N}$, $8.12 \%$.

4.10. Synthesis of (1,2,3,4-tetrakis(carbomethoxy)-1,3-pentadienyl) palladium(II)([1-(metylthio)methylene-3-(2,4,6-trimethyl)] imidazolyl-2-ene)(4a)

Complex 4a was synthesized according to published protocol [15c] (see Supplementary Material).

${ }^{1} \mathrm{HNMR}\left(\mathrm{CDCl}_{3}, \mathrm{~T}=298 \mathrm{~K}, \mathrm{ppm}\right) \delta: 2.28$ (bs, $6 \mathrm{H}, o$-aryl- $\left.\mathrm{CH}_{3}\right), 2.34$ (s, 3H, p-aryl- $\left.\mathrm{CH}_{3}\right), 2.37$ (s, 3H, S- $\left.\mathrm{CH}_{3}\right), 3.13\left(\mathrm{~s}, 3 \mathrm{H}, \mathrm{O}-\mathrm{CH}_{3}\right), 3.56$ (s, $\left.3 \mathrm{H}, \mathrm{O}-\mathrm{CH}_{3}\right), 3.59\left(\mathrm{~s}, 3 \mathrm{H}, \mathrm{O}-\mathrm{CH}_{3}\right), 3.70\left(\mathrm{~s}, 3 \mathrm{H}, \mathrm{O}-\mathrm{CH}_{3}\right), 5.05(\mathrm{bs}, 2 \mathrm{H}$, $\left.\mathrm{CH}_{2} \mathrm{~S}\right), 6.89(\mathrm{~d}, J=1.9 \mathrm{~Hz}, 1 \mathrm{H}, \mathrm{CH}=\mathrm{CH} \mathrm{Im}) 7.02$ (bs, $2 \mathrm{H}, m$-aryl-H), $7.36(\mathrm{~d}, J=1.9 \mathrm{~Hz}, 1 \mathrm{H}, \mathrm{CH}=\mathrm{CH} \mathrm{Im})$.

${ }^{13} \mathrm{C}\left\{{ }^{1} \mathrm{H}\right\}$ NMR $\left(\mathrm{CDCl}_{3}, \mathrm{~T}=298 \mathrm{~K}, \mathrm{ppm}\right) \delta: 18.2\left(\mathrm{CH}_{3}, p\right.$-mesityl$\left.\mathrm{CH}_{3}\right), 19.4\left(\mathrm{CH}_{3}\right.$, o-mesityl- $\left.\mathrm{CH}_{3}\right), 20.9\left(\mathrm{CH}_{3}, \mathrm{~S}-\mathrm{CH}_{3}\right), 50.9\left(\mathrm{CH}_{3}\right.$ $\left.\mathrm{O}-\mathrm{CH}_{3}\right), 51.1\left(\mathrm{CH}_{3}, \mathrm{O}-\mathrm{CH}_{3}\right), 51.2\left(\mathrm{CH}_{3}, \mathrm{O}-\mathrm{CH}_{3}\right), 51.3\left(\mathrm{CH}_{3}, \mathrm{O}-\mathrm{CH}_{3}\right)$, $55.3\left(\mathrm{CH}_{2}, \mathrm{SCH}_{2}\right), 120.0(\mathrm{CH}, \mathrm{CH}=\mathrm{CH} \mathrm{Im}), 129.7(\mathrm{CH}, m$-mesityl- $\mathrm{CH})$; 134.1(C, $i$-mesityl-C); 135.2 (C, o-mesityl-C); 143.8 (C, C=C), 151.8 (C, $C=C), 154.4(C, C=C), 163.6$ (C, CO), 166.6 (C, CO), 172.1 (C, CO), $175.8(\mathrm{C}, \mathrm{C}=\mathrm{C}), 176.1$ (C, CO), 186.0 (C, carbene). IR (KBr): $1695 \mathrm{~cm}^{-1}$ $\left(v_{C O}\right)$. Anal. Calcd for $\mathrm{C}_{26} \mathrm{H}_{30} \mathrm{~N}_{2} \mathrm{O}_{8}$ PdS: C, 49.02; H, 4.75; N, 4.40 . Found C, 49.12; H, 4.82; N, 4.36\%.

\subsection{Synthesis of (1,2,3,4-tetrakis(carbomethoxy)-1,3-pentadienyl)} palladium(II)([1-(2-pyridyl)methylene-3-(2,4,6-trimethyl)] imidazolyl-2-ene)(4b)

Complex $\mathbf{4 b}$ was synthesized according to published protocol [15c] (see Supplementary Material).

${ }^{1} \mathrm{H}$ NMR $\left(\mathrm{CD}_{2} \mathrm{Cl}_{2}, \mathrm{~T}=298 \mathrm{~K}, \mathrm{ppm}\right) \delta: 2.21$ (bs, 6H, o-mesityl- $\mathrm{CH}_{3}$ ), 2.37 (s, 3H, p-mesityl- $\left.\mathrm{CH}_{3}\right), 3.24\left(\mathrm{~s}, 3 \mathrm{H}, \mathrm{OCH}_{3}\right), 3.35\left(\mathrm{~s}, 3 \mathrm{H}, \mathrm{OCH}_{3}\right)$, $3.55\left(\mathrm{~s}, 3 \mathrm{H}, \mathrm{OCH}_{3}\right), 3.56\left(\mathrm{~s}, 3 \mathrm{H}, \mathrm{OCH}_{3}\right), 5.17$ (bs, $\left.1 \mathrm{H}, \mathrm{NCH}_{2}\right), 5.99$ (bs, $\left.1 \mathrm{H}, \mathrm{NCH}_{2}\right), 6.86(\mathrm{~d}, J=1.9 \mathrm{~Hz}, 1 \mathrm{H}, \mathrm{CH}=\mathrm{CH} \mathrm{Im}), 7.03(\mathrm{~s}, 2 \mathrm{H}, m-$ mesityl-H), 7.25 (d, $J=1.9 \mathrm{~Hz}, 1 \mathrm{H}, \mathrm{CH}=\mathrm{CH} \mathrm{Im}$ ), 7.42 (ddd, $J=7.7,4.6$, $1.3 \mathrm{~Hz}, 1 \mathrm{H}, 5$-Pyr); 7.60 (d, $J=7.7 \mathrm{~Hz}, 1 \mathrm{H}, 3-\mathrm{Pyr}$ ); 7.89 (td, $J=7.6$, $1.7 \mathrm{~Hz}, 1 \mathrm{H}, 4-\mathrm{Pyr}), 8.64$ (d, $J=4.6 \mathrm{~Hz}, 1 \mathrm{H}, 6-\mathrm{Pyr}) .{ }^{13} \mathrm{C}\left\{{ }^{1} \mathrm{H}\right\} \mathrm{NMR}$ $\left(\mathrm{CD}_{2} \mathrm{Cl}_{2}, \mathrm{~T}=298 \mathrm{~K}, \mathrm{ppm}\right) \delta: 19.3\left(\mathrm{CH}_{3}, o\right.$-mesityl $), 20.6\left(\mathrm{CH}_{3}, p-\right.$ mesityl), $50.0\left(\mathrm{CH}_{3}, \mathrm{COOCH}_{3}\right), 50.8\left(\mathrm{CH}_{3}, \mathrm{COOCH}_{3}\right), 50.9\left(\mathrm{CH}_{3}\right.$, $\left.\mathrm{COOCH}_{3}\right), 51.0\left(\mathrm{CH}_{3}, \mathrm{COOCH}_{3}\right), 56.3\left(\mathrm{CH}_{2}, \mathrm{CH}_{2}-\mathrm{Pyr}\right), 120.5(\mathrm{CH}, \mathrm{CH}=$ $\mathrm{CH} \mathrm{Im}), 123.6(\mathrm{CH}, \mathrm{CH}=\mathrm{CH} \mathrm{Im}), 123.9(\mathrm{CH}, 3-\mathrm{Pyr}), 124.4(\mathrm{CH}, 5-\mathrm{Pyr})$, 129.5 (CH, m-mesityl), 134.0 (C, o-mesityl), 134.8 (C, $i$-mesityl), 138.3 (C, C=C), 138.8 ( CH, 4-Pyr), 140.2 (C, p-mesityl), 149.0 (C, C= C), 152.8 ( CH, 6-Pyr), 153.9 (C, C=C), 154.4 (C, 2-Pyr), 163.4 (C, CO), 166.0 (C, CO), 173.4 (C, CO), 174.8 (C, CO), 178.3 (C, C=C), 183.0 (C, carbene). IR (KBr): $1700 \mathrm{~cm}^{-1}\left(v_{\mathrm{CO}}\right)$. Anal. Calcd for $\mathrm{C}_{30} \mathrm{H}_{31} \mathrm{~N}_{3} \mathrm{O}_{8} \mathrm{Pd}$ : C, 53.94; H, 4.68; N, 6.29. Found C, 53.84; H, 4.64; N, 6.36\%. 
4.12. Synthesis of (1,2,3,4-tetrakis(carbomethoxy)-1,3-pentadienyl) palladium(II)(8-(diphenylphosphino)quinoline) (5a)

Complex 5a was synthesized according to published protocol [17b] (see Supplementary Material).

${ }^{1} \mathrm{H}$ NMR $\left(\mathrm{CDCl}_{3}, \mathrm{~T}=298 \mathrm{~K}, \mathrm{ppm}\right) \delta: 2.77\left(\mathrm{~s}, 3 \mathrm{H}, \mathrm{OCH}_{3}\right), 3.64(\mathrm{~s}$, $\left.3 \mathrm{H}, \mathrm{OCH}_{3}\right), 3.71\left(\mathrm{~s}, 3 \mathrm{H}, \mathrm{OCH}_{3}\right), 3.73\left(\mathrm{~s}, 3 \mathrm{H}, \mathrm{OCH}_{3}\right), 7.39-7.61(\mathrm{~m}, 8 \mathrm{H}$, $\mathrm{H}^{3}, \mathrm{Ph}$ ), 7.64 (ddd, $\left.1 \mathrm{H}, J=7.7,7.2,1.2 \mathrm{~Hz}, \mathrm{H}^{6}\right), 7.66-7.77\left(\mathrm{~m}, 4 \mathrm{H}, \mathrm{H}^{5}\right.$, $\mathrm{Ph}), 7.98\left(\mathrm{~d}, 1 \mathrm{H}, J=7.9 \mathrm{~Hz}, \mathrm{H}^{7}\right), 8.37\left(\mathrm{dt}, 1 \mathrm{H}, J=8.5,1.4 \mathrm{~Hz}, \mathrm{H}^{4}\right), 9.06$ $\left(\mathrm{dd}, 1 \mathrm{H}, J=5.0,1.4 \mathrm{~Hz}, \mathrm{H}^{2}\right)$.

${ }^{31} \mathrm{P}\left\{{ }^{1} \mathrm{H}\right\} \mathrm{NMR}\left(\mathrm{CDCl}_{3}, \mathrm{~T}=298 \mathrm{~K}, \mathrm{ppm}\right) \delta: 33.4$.

${ }^{13} \mathrm{C}\left\{{ }^{1} \mathrm{H}\right\} \mathrm{NMR}\left(\mathrm{CDCl}_{3}, \mathrm{~T}=298 \mathrm{~K}, \mathrm{ppm}\right) \delta: 50.5\left(\mathrm{CH}_{3}, \mathrm{OCH}_{3}\right), 50.9$ $\left(\mathrm{CH}_{3}, \mathrm{OCH}_{3}\right), 51.2\left(\mathrm{CH}_{3}, \mathrm{OCH}_{3}\right), 51.3\left(\mathrm{CH}_{3}, \mathrm{OCH}_{3}\right), 122.6\left(\mathrm{CH}, \mathrm{C}^{3}\right), 127.8$ $\left(\mathrm{d}, \mathrm{CH}, \mathrm{J}_{\mathrm{CP}}=5.5 \mathrm{~Hz}, \mathrm{C}^{6}\right), 129.3\left(\mathrm{~d}, \mathrm{C}, \mathrm{J}_{\mathrm{CP}}=7.8 \mathrm{~Hz}, \mathrm{C}^{10}\right), 131.1\left(\mathrm{CH}, \mathrm{C}^{5}\right)$, $135.9\left(\mathrm{~d}, \mathrm{C}, \mathrm{J}_{\mathrm{CP}}=41.7 \mathrm{~Hz}, \mathrm{C}^{8}\right), 136.1\left(\mathrm{CH}, \mathrm{C}^{7}\right), 139.0\left(\mathrm{CH}, \mathrm{C}^{4}\right), 143.2(\mathrm{C}$, $\mathrm{C}=\mathrm{C}), 150.1(\mathrm{C}, \mathrm{C}=\mathrm{C}), 150.9\left(\mathrm{~d}, \mathrm{C}, \mathrm{J}_{\mathrm{CP}}=21.6 \mathrm{~Hz}, \mathrm{C}^{9}\right), 154.9\left(\mathrm{CH}, \mathrm{C}^{2}\right)$, $157.2\left(\mathrm{~d}, \mathrm{C}, \mathrm{J}_{\mathrm{CP}}=7.5 \mathrm{~Hz}, \mathrm{C}=\mathrm{C}\right), 164.1(\mathrm{C}, \mathrm{C}=\mathrm{O}), 165.3(\mathrm{C}, \mathrm{C}=\mathrm{O}), 173.1$ $\left(\mathrm{d}, \mathrm{C}, \mathrm{J}_{\mathrm{CP}}=8.0 \mathrm{~Hz}, \mathrm{C}=\mathrm{O}\right), 175.4\left(\mathrm{~d}, \mathrm{C}, \mathrm{J}_{\mathrm{CP}}=7.4 \mathrm{~Hz}, \mathrm{C}=\mathrm{O}\right), 176.7(\mathrm{C}, \mathrm{C}=$ C).

Anal. Calcd for $\mathrm{C}_{33} \mathrm{H}_{28} \mathrm{NO}_{8} \mathrm{PPd}$ : C 56.30, H 4.01, N 1.99. Found: C 56.41, H 4.18, N 1.87 .

\subsection{Synthesis of (1,2,3,4-tetrakis(carbomethoxy)-1,3-pentadienyl) palladium(II)(8-(methylthio)quinoline) (5b)}

Complex 5b was synthesized according to published protocol [17a] (see Supplementary Material).

${ }^{1} \mathrm{H} \mathrm{NMR}\left(\mathrm{CDCl}_{3}, \mathrm{~T}=298 \mathrm{~K}, \mathrm{ppm}\right) \delta: 2.87\left(\mathrm{~s}, 3 \mathrm{H}, \mathrm{SCH}_{3}\right), 3.72(\mathrm{~s}, 3 \mathrm{H}$, $\left.\mathrm{OCH}_{3}\right), 3.74\left(\mathrm{~s}, 3 \mathrm{H}, \mathrm{OCH}_{3}\right), 3.77\left(\mathrm{~s}, 3 \mathrm{H}, \mathrm{OCH}_{3}\right), 3.79\left(\mathrm{~s}, 3 \mathrm{H}, \mathrm{OCH}_{3}\right), 7.63$ $\left(\mathrm{dd}, 1 \mathrm{H}, J=8.3,5.1 \mathrm{~Hz}, \mathrm{H}^{3}\right), 7.73\left(\mathrm{dd}, 1 \mathrm{H}, J=8.2,7.3 \mathrm{~Hz}, \mathrm{H}^{6}\right), 8.01(\mathrm{~d}$, $\left.1 \mathrm{H}, J=8.2,1.3 \mathrm{~Hz}, \mathrm{H}^{5}\right), 8.11\left(\mathrm{dd}, 1 \mathrm{H}, J=7.3,1.3 \mathrm{~Hz}, \mathrm{H}^{7}\right), 8.46(\mathrm{dd}, 1 \mathrm{H}$, $\left.J=8.3,1.5 \mathrm{~Hz}, \mathrm{H}^{4}\right), 9.00\left(\mathrm{dd}, 1 \mathrm{H}, J=5.1,1.5 \mathrm{~Hz}, \mathrm{H}^{2}\right)$.

${ }^{13} \mathrm{C}\left\{{ }^{1} \mathrm{H}\right\} N M R\left(\mathrm{CDCl}_{3}, \mathrm{~T}=298 \mathrm{~K}, \mathrm{ppm}\right) \delta: 27.5\left(\mathrm{CH}_{3}, \mathrm{SCH}_{3}\right), 52.1$ $\left(\mathrm{CH}_{3}, \mathrm{OCH}_{3}\right), 51.2\left(\mathrm{CH}_{3}, \mathrm{OCH}_{3}\right), 51.4\left(\mathrm{CH}_{3}, \mathrm{OCH}_{3}\right), 51.4\left(\mathrm{CH}_{3}, \mathrm{OCH}_{3}\right)$ $122.9\left(\mathrm{CH}, \mathrm{C}^{3}\right), 128.3\left(\mathrm{CH}, \mathrm{C}^{6}\right), 130.3\left(\mathrm{C}, \mathrm{C}^{10}\right), 130.9\left(\mathrm{CH}, \mathrm{C}^{5}\right), 131.9(\mathrm{C}$, $\left.\mathrm{C}^{8}\right), 136.4\left(\mathrm{CH}, \mathrm{C}^{7}\right), 139.9\left(\mathrm{CH}, \mathrm{C}^{4}\right), 143.8(\mathrm{C}, \mathrm{C}=\mathrm{C}), 148.7(\mathrm{C}, \mathrm{C}=\mathrm{C})$, $149.0\left(\mathrm{C}, \mathrm{C}^{9}\right), 154.3\left(\mathrm{CH}, \mathrm{C}^{2}\right), 158.6(\mathrm{C}, \mathrm{C}=\mathrm{C}), 163.8(\mathrm{C}, \mathrm{C}=\mathrm{O}), 164.8(\mathrm{C}$, $C=0), 169.2(C, C=C), 173.2(C, C=0), 174.1(C, C=0)$.

IR $(\mathrm{KBr}): v_{\mathrm{C}}={ }_{\mathrm{O}} 1712,1689 \mathrm{~cm}^{-1}$

Anal. Calcd. for $\mathrm{C}_{22} \mathrm{H}_{21} \mathrm{NO}_{8} \mathrm{PdS}$ : C 46.69, $\mathrm{H}$ 3.74, N 2.48. Found: $\mathrm{C}$ 46.81, H 3.62, N 2.33.

4.14. Synthesis of (1,2,3,4-tetrakis(carbomethoxy)-1,3-pentadienyl) palladium(II)(2-((methylthio)methyl)pyridine) (6)

Complex 6 was synthesized according to published protocol [17c] (see Supplementary Material).

${ }^{1} \mathrm{H}$ NMR $\left(\mathrm{CDCl}_{3}, \mathrm{~T}=298 \mathrm{~K}, \mathrm{ppm}\right): 2.24\left(\mathrm{~s}, 3 \mathrm{H}, \mathrm{S}-\mathrm{CH}_{3}\right), 3.65(\mathrm{~s}, 3 \mathrm{H}$ $\left.\mathrm{COOCH}_{3}\right), 3.70\left(\mathrm{~s}, 3 \mathrm{H}, \mathrm{COOCH}_{3}\right), 3.72\left(\mathrm{~s}, 6 \mathrm{H}, \mathrm{COOCH}_{3}\right), 4.11(\mathrm{~s}, 2 \mathrm{H}$, pyr- $\left.\mathrm{CH}_{2} \mathrm{~S}\right), 7.41\left(\mathrm{td}, 1 \mathrm{H}, \mathrm{H}^{5} \mathrm{pyr}, \mathrm{J} 1=7.5 \mathrm{~Hz}, \mathrm{~J} 2=1.4 \mathrm{~Hz}\right), 7.5\left(\mathrm{~d}, 1 \mathrm{H}, \mathrm{H}^{3}\right.$ pyr, $J=7.7 \mathrm{~Hz}$ ), $7.88\left(\mathrm{td}, 1 \mathrm{H}, \mathrm{H}^{4} \mathrm{pyr}, \mathrm{J} 1=7.5 \mathrm{~Hz}, \mathrm{~J} 2=1.7 \mathrm{~Hz}\right), 8.65(\mathrm{dd}$, $\left.1 \mathrm{H}, \mathrm{H}^{6} \mathrm{pyr}, J=5.6 \mathrm{~Hz}\right) .{ }^{13} \mathrm{C} \mathrm{NMR}\left(\mathrm{CDCl}_{3}, T=298 \mathrm{~K}, \mathrm{ppm}\right): 18.6$ $\left(\mathrm{S}-\mathrm{CH}_{3}\right), 43.4\left(\right.$ pyr- $\left.\mathrm{CH}_{2}-\mathrm{S}\right), 51.5\left(\mathrm{COOCH}_{3}\right), 51.8\left(\mathrm{COOCH}_{3}\right), 51.9$ $\left(\mathrm{COOCH}_{3}\right), 124.7\left(\mathrm{C}^{5} \mathrm{pyr}\right), 125.0\left(\mathrm{C}^{3} \mathrm{pyr}\right), 139.7\left(\mathrm{C}^{4} \mathrm{pyr}\right), 152.5\left(\mathrm{C}^{6}\right.$ pyr $), 160.0$ ( $\mathrm{C}^{2}$ pyr $), 144.4(\mathrm{C}=\mathrm{C}), 149.0(\mathrm{C}=\mathrm{C}), 158.1(\mathrm{C}=\mathrm{C}), 169.2$ $(\mathrm{C}=\mathrm{C}), 164.1\left(\mathrm{COOCH}_{3}\right), 165.3\left(\mathrm{COOCH}_{3}\right), 173.7\left(\mathrm{COOCH}_{3}\right), 173.9$ $\left(\mathrm{COOCH}_{3}\right)$. Anal. Calcd for C19H21 NO8PdS: C 43.07, H 3.99, N 2.64. Found: C 42.98, H 3.96, N 2.59.

\subsection{Crystal structure determination}

The crystal data of 1a were collected at $100 \mathrm{~K}$ at the XRD1 beamline of the Elettra Synchrotron, Trieste (Italy) [23], using a monochromatic wavelength of $0.700 \AA$. The data sets were integrated and corrected for Lorentz, absorption and polarization effects with the XDS package [24]. The structures were solved by direct methods using SHELXT program [25] and refined using full-matrix least-squares implemented in SHELXL-2018/3 [26]. Thermal motions for all non-hydrogen atoms were treated anisotropically and hydrogens have been included on calculated positions, riding on their carrier atoms. Geometric and thermal restraints (DFIX, DANG and SIMU) were applied to ligands disordered sidechains. No solvent molecules were found in the crystal packing. The Coot program was used for structure building [27]. The crystal data are given in Table S1. Pictures were prepared using Ortep3 [28] and Pymol [29] softwares.

Crystallographic data has been deposited at the Cambridge Crystallographic Data Centre and allocated the deposition number CCDC 1911788. These data can be obtained free of charge via https://www.ccdc.cam.ac.uk/structures.

\subsection{Cell viability assays}

Cells were grown in accordance with the supplier and maintained at $37{ }^{\circ} \mathrm{C}$ in a humidified atmosphere of $5 \%$ carbon dioxide. Five hundred cells were plated in 96 wells and grown for $96 \mathrm{~h}$. Cell viability was measured with CellTiter glow assay (Promega, Madison, WI, USA) with a Tecan M1000 instrument. IC $_{50}$ values were calculated from logistical dose response curves. Averages were obtained from triplicates and error bars are standard deviations.

\subsection{Immunofluorescence microscopy analyses}

Cells were seeded at a density of $2 \times 10^{5}$ cells/mL on coverslip glass (pretreated with poly-D-Lysine, $1 \mu \mathrm{g} / \mathrm{mL}$ ) inserted in 6 -well plates. After overnight culture at $37{ }^{\circ} \mathrm{C}$ and $5 \% \mathrm{CO}_{2}$, cells were treated with different concentrations of compound 1a $(40,80$, $320 \mathrm{~nm}$ or $1 \mu \mathrm{M})$ or cisplatin $(10 \mu \mathrm{M})$ for 3,6 , and $12 \mathrm{~h}$. Cells were fixed in $4 \%$ paraformaldehyde/PBS ( $20 \mathrm{~min}$, RT), permeabilized with $0.3 \%$ Triton X-100/PBS (15 min, RT) and blocked in 8\% BSA/PBS ( $1 \mathrm{~h}$, RT). Cells were stained with mouse monoclonal anti-phosphohistone pS139H2A.X antibody (1:100 dilution in 1\% BSA/PBS, at $4{ }^{\circ} \mathrm{C}, \mathrm{ON}$ ) or with mouse monoclonal anti-cytochrome $\mathrm{c}$ antibody ( $1: 100$ dilution in $1 \% \mathrm{BSA} / \mathrm{PBS}$, at $4{ }^{\circ} \mathrm{C}$, ON) which were obtained respectively from Millipore (Cat. \# 05-636; Burlington, MA, USA) and Cell Signaling Technology (Cat. \# 12963; Danvers, MA, USA), and labeled with secondary antibodies (Alexa Fluor ${ }^{\circledR} 488$ dye, 1:1000 dilution, RT, 2 h) obtained from Cell Signaling Technology (Cat. \# 4408; Danvers, MA, US). To visualize DNA, cells were stained with DAPI $1 \mathrm{mg} / \mu \mathrm{L}$ (1:1000 dilution in PBS, at $\left.4{ }^{\circ} \mathrm{C}, 1 \mathrm{~min}\right)$. Cells were washed three times with $1 \times$ PBS after all incubations. All the coverslips were mounted in fluorSave ${ }^{\mathrm{TM}}$ reagent (Cat. \# 345789; Millipore: Burlington, MA, USA), the cells were examined with a Nikon Eclipse Ti fluorescence microscope, and the images were analyzed with NIS Elements software version 3.0 (Nikon: Shinagawa, Tokyo, Japan).

\subsection{Flow cytometry analysis}

Cells were seeded at a density of $10^{5}$ cells $/ \mathrm{mL}$ on $10 \mathrm{~mL}$ plates. After incubation overnight at $37^{\circ} \mathrm{C}$ in $5 \% \mathrm{CO}_{2}$, cells were treated with different concentrations of compound 1a $(40,80$ or $320 \mathrm{nM})$ or cisplatin $(10 \mu \mathrm{M})$ for 24,48 and $96 \mathrm{~h}$. Cells were collected by trypsinization and centrifugation for each time point. After a washing step with $1 \times \mathrm{PBS}$, the pellet was resuspended in $1 \mathrm{~mL}$ of PBS and $9 \mathrm{~mL}$ of cold $70 \%$ EtOH while vortexing. $10^{6}$ cells were incubated in $70 \% \mathrm{EtOH}$ at $4{ }^{\circ} \mathrm{C}$ for at least $2 \mathrm{~h}$. Cells were re-collected by centrifugation and suspended in $300 \mu$ of the propidium iodide/ RNAse A fresh solution (PBS, $10 \mathrm{mg} / \mathrm{mL}$ RNase A, $1 \mathrm{mg} / \mathrm{mL}$ propidium iodide). Cells were incubated at $37^{\circ} \mathrm{C}$ for $60 \mathrm{~min}$ then 
analyzed by a FACSCalibur flow cytometer (BD Biosciences, Franklin Lakes, NJ, USA). Populations of cells at different phases of cell cycle were analyzed with the ModFit LT4.0 software.

\subsection{Caspase-3/7 activity detection}

The activity of caspase-3/7 was determined by Caspase-Glo ${ }^{\mathrm{TM}} 3$ / 7 Assay (Cat. \#G8091; Promega: Madison, WI, USA). Cells were seeded at a density of $10^{5}$ cells $/ \mathrm{mL}$ on 6 -well plates. After incubation overnight at $37^{\circ} \mathrm{C}$ in $5 \% \mathrm{CO}_{2}$, cells were treated with different concentrations of compound 1a $(40,80,320 \mathrm{nM}$ or $1 \mu \mathrm{M})$ or cisplatin $(10 \mu \mathrm{M})$ for 3,12 , and $24 \mathrm{~h}$. Cells were collected by trypsinization and centrifugation. Cells were lysed in a buffer containing Tris $\mathrm{HCl} 1 \mathrm{M} \mathrm{pH} 8, \mathrm{NaCl} 5 \mathrm{M}, 10 \%$ glycerol, 1\% NP-40, EDTA $0.5 \mathrm{M}$, sodium orthovanadate $2 \mathrm{mM}$, sodium fluoride $5 \mathrm{mM}$ and EDTA-free protease inhibitors 25× (Cat. \# 04693132001, Roche: Basel, Switzerland). The cellular protein content was quantified with an Eppendorf BioPhotometer (Hamburg, Germany). The assay was performed with $40 \mu \mathrm{g}$ of protein extracts in 1:1 ratio of CaspaseGlo $^{\circledR} 3 / 7$ reagent in triplicate in 96 -well plate. The assay was incubated at RT for 30min. Luminescence was recorded using a Tecan M1000 PRO instrument (Männedorf, Zürich, Switzerland).

\section{Acknowledgments}

FR and IC are thankful to AIRC (My First AIRC, 15639).

\section{Appendix A. Supplementary data}

Supplementary data to this article can be found online at https://doi.org/10.1016/j.ejmech.2019.06.065.

\section{References}

[1] (a) L. Kelland, Nat. Rev. Canc. 7 (2007) 573-584;

(b) M.A. Fuentes, C. Alonso, J.M. Perez, Chem. Rev. 103 (2003) 645-662;

(c) Y. Jung, S.J. Lippard, Chem. Rev. 107 (2007) 1387-1407;

(d) P.J. O'Dwyer, J.P. Stevenson, S.W. Johnson, in: B. Lippert (Ed.), Cisplatin: Chemistry and Biochemistry of a Leading Anticancer Drug, Wiley-VCH, Weinheim, 1999.

[2] (a) R. Oun, Y.E. Moussa, N.J. Wheate, Dalton Trans. 47 (2018) 6645-6653; (b) N.J. Wheate, S. Walker, G.E. Craig, R. Oun, Dalton Trans. 39 (2010) 8113-8127.

[3] (a) M.J. Clarke, F. Zhu, D.R. Frasca, Chem. Rev. 99 (1999) 2511-2534; (b) S. Komeda, A. Casini, Curr. Top. Med. Chem. 12 (2012) 219-235.

[4] M.D. Coskun, F. Ari, A.Y. Oral, Bioorg. Med. Chem. 21 (2013) 4698-4705.

[5] (a) M.M. Shoukry, A.A. Shoukry, M.N. Hafez, J. Coord. Chem. 63 (2010) 652-664;

(b) H. Hohamann, R. van Eldik, Inorg. Chim. Acta 174 (1990) 87-92;

(c) H. Hohamann, H. Suvachittanont, R. van Eldik, Inorg. Chim. Acta 177 (1990) $51-58$.

[6] A.S. Abu-Surrah, M. Kettunen, Curr. Med. Chem. 13 (2006) 1337-1357.

[7] (a) S. Ray, R. Mohan, J.K. Singh, M.K. Samantaray, M.M. Shaikh, D. Panda, P. Ghosh. J. Am. Chem. Soc. 129 (2007) 15042-15053;

(b) N. Cutillas, G.S. Yellol, C. de Haro, C. Vicente, V. Rodríguez, J. Ruiz, Coord. Chem. Rev. 257 (2013) 2784-2797;

(c) A.R. Kapdi, I.J.S. Fairlamb, Chem. Soc. Rev. 43 (2014) 4751-4777;

(d) S. Medici, M. Peana, V.M. Nurchi, J.I. Lachowicz, G. Crisponi, M.A. Zoroddua, Coord. Chem. Rev. 284 (2015) 329-350;

(e) T.H. Fong, C.N. Lok, C.Y.S. Chung, Y.M.E. Fong, P.K. Chow, P.K. Wan, C.M. Che, Angew. Chem. Int. Ed. 55 (2016) 11935-11939.

[8] A.J. Arduengo, R.L. Harlow, M. Kline, J. Am. Chem. Soc. 113 (1991) 361-363.
[9] (a) M. Scholl, T.M. Trnka, J.P. Morgan, R.H. Grubbs, Tetrahedron Lett. 40 (1999) 2247-2250

(b) J. Huang, H.Z. Schanz, E.D. Stevensen, S.P. Nolan, Organometallics 18 (1999) 5375-5380;

(c) W.A. Hermann, M. Elison, J. Fischer, C. Köcher, G.R.J. Artus, Angew Chem. Int. Ed. Engl. 34 (1995) 2371-2374.

[10] (a) W. Li, R. Gust, Chem. Soc. Rev. 42 (2013) 755-773;

(b) I. Ott, I. Medicinal Chemistry of Metal N-Heterocyclic Carbene Complexes (Book Chapter of Inorganic and Organometallic Transition Metal Complexes with Biological Molecules and Living Cells), 2017;

(c) W.A. Hermann, Angew. Chem. Int. Ed. 41 (2002) 1290-1309;

(d) L. Cavallo, A. Correa, C. Costabile, H. Jacobsen, J. Organomet. Chem. 690 (2005) 5407-5413, 690;

(e) S. Díez-González, S.P. Nolan, Coord. Chem. Rev. 251 (2007) 874-883;

(f) P. de Frémont, N. Marion, S.P. Nolan, Coord. Chem. Rev. 253 (2009) 862-892.

[11] K.M. Hindi, M.J. Panzner, C.A. Tessier, C.L. Cannon, W.J. Youngs, Chem. Rev. 109 (2009) 3859-3884.

[12] (a) T. Scattolin, I. Caligiuri, L. Canovese, N. Demitri, R. Gambari, I. Lampronti, F. Rizzolio, C. Santo, F. Visentin, Dalton Trans, 47 (2018) 13616-13630;

(b) T. Scattolin, S. Giust, P. Bergamini, I. Caligiuri, L. Canovese, N. Demitri, R. Gambari, I. Lampronti, F. Rizzolio, F. Visentin, Appl. Organomet. Chem. 33 (2019), e4902.

[13] (a) B.M. Trost, Acc. Chem. Res. 35 (2002) 695-705;

(b) F. Visentin, A. Togni, Organometallics 26 (2007) 3746-3754;

(c) L. Canovese, F. Visentin, T. Scattolin, C. Santo, V. Bertolasi, Polyhedron 119 (2016) 377-386;

(d) R. Van Belzen, C.J. Elsevier, A. Didieu, N. Veldman, A.L. Speck, Organometallics 22 (2003) 722-736.

[14] A. Heumann, Transition Metals for Organic Synthesis, vol 1, Wiley-VCH, 2004 chap. 2.14 .

[15] (a) L. Canovese, F. Visentin, T. Scattolin, C. Santo, V. Bertolasi, J. Organomet. Chem. 808 (2016) 48-56;

(b) T. Scattolin, F. Visentin, C. Santo, V. Bertolasi, L. Canovese, Dalton Trans. 45 (2016) 11560-11567;

(c) L. Canovese, C. Santo, T. Scattolin, F. Visentin, V. Bertolasi, J. Organomet Chem. 794 (2015) 288-300;

(d) F. Visentin, C. Santo, T. Scattolin, N. Demitri, L. Canovese, Dalton Trans, 46 (2017) 10399-10407.

[16] (a) M.G. Gardiner, C.C. Ho, Coord. Chem. Rev. 375 (2018) 373-388;

(b) M. Nirmala, G. Saranya, P. Viswanathamurthi, R. Bertani, P. Sgarbossa, J.G. Malecki, J. Organomet. Chem. 831 (2017) 1-10;

(c) D. Munz, C. Allolio, D. Meyer, M. Micksch, L. Roessner, T. Strassner, J. Organomet. Chem. 794 (2015) 330-335;

(d) M.V. Baker, D.H. Brown, P.V. Simpson, B.W. Skelton, A.H. White, C.C. Williams, J. Organomet. Chem. 691 (2006) 5845-5855;

(e) A. Biffis, M. Cipani, E. Bressan, C. Tubaro, C. Graiff, A. Venzo, Organometallics 33 (2014) 2182-2188.

[17] (a) L. Canovese, F. Visentin, T. Scattolin, C. Santo, V. Bertolasi, Polyhedron 113 (2016) 25-34;

(b) L. Canovese, F. Visentin, T. Scattolin, C. Santo, V. Bertolasi, Dalton Trans. 44 (2015) 15049-15058;

(c) L. Canovese, F. Visentin, G. Chessa, P. Uguagliati, C. Levi, A. Dolmella, Organometallics 24 (2005), 5537-5348.

[18] T. Scattolin, L. Canovese, F. Visentin, C. Santo, N. Demitri, Polyhedron 154 (2018) 382-389.

[19] K. Moseley, P.M. Maitlis, J. Chem. Soc. Dalton Trans. (1974) 169-175.

[20] P. Zhang, P.J. Sadler, J. Organomet. Chem. 839 (2017) 5-14.

[21] J. Yuan, R. Adamski, J. Chen, FEBS (Fed. Eur. Biochem. Soc.) Lett. 584 (2010) 3717-3724.

[22] S. Fulda, K.M. Debatin, Oncogene 25 (2006) 4798-4811.

[23] A. Lausi, M. Polentarutti, S. Onesti, J.R. Plaisier, E. Busetto, G. Bais, L. Barba, A. Cassetta, G. Campi, D. Lamba, A. Pifferi, S.C. Mande, D.D. Sarma, S.M. Sharma, G. Paolucci, Eur. Phys. J. Plus 130 (2015) 1-8.

[24] W. Kabsch, Acta Crystallogr. D 66 (2010) 125-132.

[25] G.M. Sheldrick, Acta Crystallogr. A 71 (2015) 3-8.

[26] G.M. Sheldrick, Acta Crystallogr. C 71 (2015) 3-8.

[27] P. Emsley, B. Scott, W. Lohkamp, K. Cowtan, Acta Crystallogr. D 66 (2010) 486-501.

[28] L. Farrugia, J. Appl. Crystallogr. 45 (2012) 849-854.

[29] L. Schrödinger, The PyMOL molecular graphics system. http://www.pymol. org, 2015. 